\title{
Austeritätspolitik: Was Parteien bewirken und Märkte mögen
}

Klaus Armingeon

\section{Einleitung}

Das Werk von Manfred Schmidt ist ungewöhnlich breit und umfangreich. Es behandelt viele Politikfelder, insbesondere die Militär-, Sozial-, Wirtschafts-, Arbeitsmarkt-und Bildungspolitik. Schmidt prüft darüber hinaus, ob demokratische Institutionen und Akteure einen Unterschied für die Lebensbedingungen der Bürgerinnen und Bürger machen. Dabei richtet sich in den letzten Jahren sein Interesse auch auf den Unterschied der Leistungsprofile von Autokratien und Demokratien. Schließlich analysiert Schmidt Demokratien nicht nur empirisch. Vielmehr arbeitet er auch Theorien über Demokratien auf, vergleicht sie sorgsam miteinander und identifiziert ihre Vorzüge und Nachteile.

Einige Argumente und Befunde ziehen sich durch aus Gesamtwerk hindurch. Der Handlungsspielraum demokratischer Politik sei vielfach beschränkt und erheblich geringer, als Politiker dies im Wahlkampf behaupten. Dies sei ganz besonders auf vorhandene Ressourcen und Handlungsnotwendigkeiten zurückzuführen. Falls ein Land einen generösen Sozialstaat anstrebe, müsste hierfür erst einmal die wirtschaftliche Leistungsfähigkeit gegeben sein, die es erlaube, dessen Kosten zu tragen. Ferner müssten politische Systeme eine Reihe von funktionalen Voraussetzungen moderner Gesellschaften herstellen. Dazu zähle beispielsweise ein Alterssicherungssystem, das die Lücken decken kann, welche die Industrialisierung in die familiären und lokalen Selbsthilfenetzwerke geschlagen hat. Dies ist ein erster, zentraler Befund.

Ein zweiter zentraler Befund bezieht sich auf ,Gegenregierungen', die die Macht zentraler Regierungen einschränkten. Hierzu zählten unabhängige Zentralbanken, Landesregierungen, korporatistische Selbstregulierungen der Sozialpartner oder die Mitgliedschaft in der EU. Alle diese Gegenregierungen oder Vetopunkte begrenzten den Handlungsspielraum nationaler Regierungen.

Jede Analyse staatlicher Politiken müsse den Einfluss dieser sozio-ökonomischen und institutionellen Variablen berücksichtigen und zudem die Begrenzungen beachten, die auf die Trägheit staatlicher Politiken (,Path dependency') und auf die Wirkung internationaler Organisationen und Märkte zurückgehen. Erst wenn diese Bedingungen staatlicher Politik systematisch einbezogen werden, lasse sich etwas darüber aussagen, wie gut politische 
Parteien den verbliebenen Handlungsspielraum genutzt haben. Für diesen Test seien die Wirtschafts- und Sozialpolitiken ganz besonders gut geeignete Politikfelder, weil die Programme der großen europäischen Parteien sich vordringlich darauf beziehen.

Die ,große Rezession’ oder ,Krise’, die 2007 als Finanzkrise beginnt, 2008 die Realwirtschaft erreicht und 2010 in die europäische Staatsschuldenkrise mündet ist besonders gut geeignet, um die analytische Perspektive von Manfred Schmidt erneut empirisch auf Ergiebigkeit zu prüfen.

Die Krise taugt deshalb so gut, weil wir in ihr besonders starke Effekte des Parteienwettbewerbs erwarten. In einer bahnbrechenden Studie identifizierte Peter Gourevitch für die vorhergehenden großen Krisen in der westlichen Welt - die Krise am Ende des 19. Jahrhunderts, die Krise der 1930er Jahre und die krisenhafte Periode nach dem Herbst 1973 enorme Gestaltungschancen der politischen Akteure, weil bisherige politische Koalitionen in den Umbruchsituationen durcheinandergewirbelt wurden und neue Ideen sich in neuen politischen Konstellationen politikwirksam durchsetzen konnten (Gourevitch 1986). Frank Castles, der mit Schmidt über viele Jahre zusammen arbeitete und mit ihm zusammen ein einflussreiches Projekt über ,Do Parties Matter' leitete, sprach von Krisen als seltenen Ereignissen -schwarze Schwäne- , die Politiker nicht erwarten würden (Castles 2010). Wenn überhaupt, dann müssten unter diesen Bedingungen bedeutsame Effekte des Parteienwettbewerbs feststellbar sein - und es war nicht zuletzt Schmidt, der auf die große demokratietheoretische Relevanz dieses signifikanten Parteieneffekts hingewiesen hat. Schließlich liegt der westlichen Repräsentativdemokratie die Überlegung zugrunde, dass gesellschaftliche Interessen durch Parteien repräsentiert werden und diese Politik im Interesse ihrer Wähler und Wählerinnen betreiben.

Schmidt bemüht sich vorbildlich, seine eigenen politischen Positionen in der Analyse zu kontrollieren und Argumente aller Seiten sorgfältig und rational abzuwägen. Dennoch wird deutlich, dass der Wohlfahrtsstaat Gegenstand seiner Sorgen und seiner Bewunderung ist. Er bewundert den Wohlfahrtsstaat, weil er einen Beitrag zur Stabilisierung der Demokratie geleistet sowie soziales Elend und Armut wirksam verhindert hat. Gerade in seinen jüngsten Arbeiten (Schmidt 2012) unterstreicht Schmidt die Leistungsfähigkeit des deutschen Wohlfahrtsstaates. Er böte weit mehr, als Esping-Andersen bestenfalls dem von ihm als konservativ und statussichernden beschriebenen Sozialstaat in der Bundesrepublik Deutschland zuschreiben wollte (Esping-Andersen 1990). Auf der anderen Seite bekümmert ihn die Soll-Seite der Bilanz. Dazu gehört vor allem die Tendenz des Wohlfahrtsstaats, die Kosten auf nachfolgende Generationen über Verschuldung zu externalisieren und das 
Bildungssystem zu vernachlässigen, weil die staatlichen Ressourcen weitgehend für soziale Sicherheit gebunden sind. Aus diesem Grund sind kontinuierliche Reformen des Wohlfahrtsstaates notwendig. Diese treffen jedoch, wie dies beispielsweise Paul Pierson gezeigt hat, auf massiven Widerstand aller jener, die vom bisherigen Wohlfahrtsstaat profitieren (Pierson 1994.) Die Krise könnte nun eine Chance sein, Reformen durchzusetzen, die in normalen Zeiten kaum greifbar sind. Dazu passt die Äußerung eines engen Mitarbeiters des US-Präsidenten Obama:

,You never want a serious crisis to go to waste...Things that we have postponed too long, that were long-term, are now immediate and must be dealt with. This crisis provides the opportunity for us to do things that you could not do before.' (Wall Street Journal, Nov. 21, 2008, http://online.wsj.com/article/SB122721278056345271.html, letzter Zugriff am 13. August 2012).

Tatsächlich variierten die Wirtschafts- und Fiskalpolitiken der etablierten Demokratien zu Beginn der großen Rezession beträchtlich. Deutete dies auf eine Phase der Reform, Innovation und des mutigen Experiments hin? Länder wie Spanien und die USA schienen zunächst zum Keynesianismus zurückzukehren. Deutschland und die Schweiz beschritten einen ,mittleren Weg' zwischen einer massiven anti-zyklischen Politik und einer Austeritätspolitik, während eine dritte Ländergruppe Sparpolitiken implementierte (Armingeon 2012; Cameron 2012; Lindvall 2012). Dies änderte sich freilich rasch. Um 2010 begannen fast alle anti-zyklisch arbeitenden Länder die fiskalpolitische Expansionsstrategie zu beenden und konvergierten auf eine Strategie der angestrebten fiskalischen Konsolidierung.

Drei Fragen leiten diesen Beitrag: (1) Gab es tiefgreifende sozial- und wirtschaftspolitische Reformen seit 2010, die mit der Vergangenheit brachen und innovative Sozial- und Wirtschaftspolitiken einführten? (2) Machten politische Parteien im Zuge der fiskalpolitischen Konsolidierung einen Unterschied oder waren die Änderungen der Staatstätigkeiten nur eine Funktion des Gewichts der fiskalischen und wirtschaftlichen Probleme? (3) Vorausgesetzt, die schiere Größe der fiskalischen und ökonomischen Probleme - wie beispielsweise die Zinssätze, die Regierungen für ihre Schulden zahlen müssen - sei der zentrale Erklärungsgrund für die Reformen, wie können wir dann erklären, dass einige Länder unter sonst gleichen wirtschaftlichen Bedingungen mit so viel mehr Herausforderungen zu kämpfen hatten als andere? Gibt es vielleicht auch rein politische Gründe, weshalb die Akteure auf den Finanzmärkten die Zinsen auf Staatsanleihen für einige Nationen viel höher ansetzten, als in anderen - selbst nachdem man den Einfluss objektiver ökonomischer Faktoren kontrolliert hat? 
Ich möchte drei Befunde präsentieren. Die ersten beiden sind mit Schmidts analytischer Perspektive gut vereinbar. Freilich unterstreiche ich, dass der Handlungsspielraum der Parteien in der Krise noch kleiner war, als dies Schmidts These eines systematischen aber auch begrenzten Parteieneffekts behauptet. Der erste Befund lautet, dass die gegenwärtige Krise nicht zur Politikinnovation genutzt wurde - mit Ausnahme eines massiven wohlfahrtsstaatlichen Abbaus in den südeuropäischen Ländern, der weit über eine inkrementale Anpassung hinausgeht. Austeritätspolitik ist inzwischen zum einzigen fiskalpolitischen Programm in den wirtschaftlich hoch entwickelten Demokratien geworden. Nur unter besonders günstigen Umständen - und dies ist der zweite Befund - wirkt sich der Parteienwettbewerb auf die konkrete Umsetzung der Austeritätspolitik auf. Der dritte Befund weist auf eine Blindstelle bei Schmidt und vielen anderen Forschern im Bereich der politischen Ökonomie hin. Es wird implizit angenommen, ökonomische Prozesse verliefen weitgehend rational ab; Marktteilnehmer ließen sich von vernünftigen Kosten-NutzenKalkülen leiten und seien dabei immun gegenüber politischen Vorlieben und Ideologien. Meine Datenauswertungen zeigen jedoch eine massive Überschätzung der analytischen Leistungsfähigkeit von Finanzmärkten, weil Ideologien und politische Präferenzen der Eliten auf den Finanzmärkten auch nach Kontrolle objektiver ökonomischer Variablen auf die Marktentscheidungen durchschlagen - mit fatalen Konsequenzen für die nationalen Demokratien.

Diese Befunde können unser Wissen in dreierlei Weise ergänzen. (1) Krisen öffnen nicht umstandslos ein ,window of opportunities'. Das ist nur der Fall, wenn es politische Ideen über alternative Politiken gibt (Kingdon 1984)- beispielsweise Ideen über wirtschaftliche Öffnung oder Schließung im ausgehenden 19. Jahrhundert, Ideen über einen umfassenden Wohlfahrtsstaat und Sozialpartnerschaft versus Ideen über einen minimalistischen Sozialstaat und konfliktuelle kollektiven Arbeitsbeziehungen wie in den 1930er Jahren. 2010 gab es jedoch in den etablierten Demokratien sehr wenige programmatische Differenzen zwischen linken und rechten Parteien über makro-ökonomische oder wohlfahrtsstaatliche Politiken. Deshalb kann es keine großen Innovationen geben, da keine unterschiedlichen und neuen programmatischen Ideen existierten. (2) Manfred Schmidt hat immer wieder darauf hingewiesen, dass der Parteienwettbewerb nur dann zu unterschiedlichen Politiken führen könne, wenn es tatsächlich einen politischen Handlungskorridor gibt. Wenn die Akteure überzeugt sind, die von außen kommenden Zwangslagen hätten alle Optionen beseitigt, erwarten wir keine Varianz der Politiken. In der großen Rezession gab es kaum Ressourcen für neue Politiken und es gab starke Interessen an 
der inkrementellen Fortschreibung des bisherigen wohlfahrtsstaatlichen Regimes. Deshalb erstaunt das Ausbleiben von großen Reformen - vor allem außerhalb der besonders krisengeschüttelten südeuropäischen Länder - nicht. (3) Schließlich problematisiere ich die selbstverständliche Annahme vieler Studien, Marktakteure ließen sich nur durch ein ökonomisches Kalkül leiten. Manfred Schmidts Schüler Herbert Obinger hat überzeugend nachgewiesen, dass sich zwischen linken und rechten Regierungen oder zwischen großen und kleinen Wohlfahrtsstaaten keine bemerkenswerten Unterschiede der wirtschaftlichen Wachstumsraten beobachten lassen (Obinger 2004; siehe auch Pontusson 2005; Sala-i-Martin 2002; Hall 2001). Dennoch, so möchte ich zeigen, bestrafen die Eliten auf Finanzmärkten Länder mit linken oder zentristischen Regierungen und Nationen mit einem großen oder wachsenden Staatssektor mit höheren Zinsen auf Staatsanleihen. Diese Zinssätze sind ausgezeichnete Prädiktoren für das Ausmaß der nationalen Austeritätspolitik - und diese Sparmaßnahmen verursachen viele Härten und Leid.

Manfred Schmidt hat mich in die vergleichende Policy-Analyse eingeführt. Die Methode des Vergleichs wende ich auch hier an. Ich analysiere die 27 Demokratien der EU und die anderen etablierten Demokratien der OECD (Australien, Island, Japan, Kanada, Neuseeland, Norwegen, USA und die Schweiz). Aus Gründen der Datenverfügbarkeit können allerdings einige Analysen nicht immer für alle dieser 35 Nationalstaaten durchgeführt werden.

\section{Die ausgebliebene Politikinnovation}

Konnten nationale Regierungen in der großen Rezession neue Wege in der Wirtschafts- und Sozialpolitik beschreiten? Gelang die Modernisierung des Wohlfahrtsstaates, welche ihn nachhaltig machen kann? Konnten beispielsweise Systeme der Alterssicherung so umgebaut werden, dass sie weiterhin soziale Sicherheit liefern und gleichzeitig langfristig finanzierbar sind? Gelang es, die sogenannten ,neuen sozialen Risikogruppen’ abzusichern? Dazu gehören beispielsweise Frauen, die Beruf und Karriere vereinbaren wollen, alleinerziehende Mütter ohne Berufsausbildung oder arbeitslose Jugendliche, die weder die Kenntnisse noch die kognitiven Ressourcen haben, um in der wissensbasierten Dienstleistungsgesellschaft mithalten zu können (Armingeon and Bonoli 2006). Wurde der Wohlfahrtsstaat modernisiert, so dass Ressourcen in die neuen Sozialpolitiken umgeschichtet wurde? Oder gab es sonst Anzeichen für weitreichende qualitative Änderungen der Sozialpolitik? 
Während der Krise wurden in vielen Ländern die Systeme der Alterssicherung reformiert. Zwischen 2007 und 2010 haben mindestens 15 Länder der untersuchten Ländergruppe das Rentenalter angehoben ${ }^{1}$. Es ist freilich unklar, ob die Krise der Reformauslöser war. Die Fondazione Rodolfo DeBenedetti (http://www.frdb.org/) sammelte Daten über kostenreduzierenden Rentenreformen in 14 Ländern für den Zeitraum von 1980 bis 2009. Diese Datenbasis haben wir im Rahmen eines Forschungsprojektes bis 2011 aufdatiert $^{2}$. Es gibt keine deutliche Zunahme der Reformhäufigkeit im Zeitraum 2007-2011 im Vergleich zu den Jahren von 2000 bis 2006. Deshalb spricht vieles für die Annahme, die Finanzierungs- und Demographieprobleme der Rentensysteme hätten auch ohne Zutun der Krise diese Reformen ausgelöst.

Derselbe Befund ergibt eine Auswertung der Reformen in der aktiven Arbeitsmarktpolitik, welche die Beschäftigungschancen von Arbeitnehmern verbessern sollen. Solche Reformen wurden vielfach während der Krise verfolgt (vgl. die Übersichten der ILO und World Bank 2012). Vergleicht man jedoch die Zahl der Reformen zugunsten einer stärkeren aktiven Arbeitsmarktpolitik vor (2000-2006) und während der Krise lässt sich keine Beschleunigung des Reformtempos seit 2007 feststellen. $^{3}$

Ähnliches ergibt eine Re-Analyse einer Liste von sozio-ökonomischen Reformen in der OECD-Ländergruppe. Die OECD schlägt jedem Mitgliedsland aufgrund einer Länderanalyse strukturelle Reformen vor. Diese Politiken sollen die wirtschaftliche Effizienz erhöhen. Aus Sicht des OECD-Sekretariats eigenen sich hierzu besonders markt-freundliche (,neo-liberale') Politiken. Dazu gehören beispielsweise Wettbewerbspolitiken oder Reformen, die die neuen sozialen Risiken betreffen, da viele dieser Politiken sowohl wirtschaftliche Effizienzssteigerung als auch eine Erhöhung der Erwerbsquote anzielen. In der jüngsten OECD-Studie ‘Going for Growth’ berichtet die OECD über ihre seit 2007 vorgeschlagenen Reformen und deren Umsetzung (OECD 2012: 51-133). Im jedem Jahr ihrer Länderbeobachtung suchten sich die OECD-Experten drei Politikprioritäten aufgrund internationale vergleichbarer Indikatoren der Politiken und ihrer Wirkungen aus. Hinzu kamen zwei weitere Reformprioritäten, die 'are often supported by indicator- based evidence, but may draw principally on country-specific expertise. These priorities are meant to capture any potential policy imperatives in fields not covered by indicators' (OECD 2012: 49).

Ich kodierte jedes Set von Politiken, das sich auf diese Reformvorschläge bezieht, ob es sich um eine Politik zur Deckung neuer sozialer Risiken oder ob es sich ausschließlich um

\footnotetext{
${ }^{1}$ Zusammengestellt aus IMF 2010b: 73-74 und weiteren nationalen Quellen.

${ }^{2}$ Ich danke Rafael Labanino von der Universität Bern für diese Arbeit.

${ }^{3}$ Auch dieser Befund stützt sich auf die FRDB-Datenbank und ihrer Aufdatierungen.
} 
einer liberalisierende (markt-befördernde) Politik handelte. Anschließend erfasste ich die Anzahl der realisierten Politiken. Die Analyse konnte für 29 Länder durchgeführt werden. Im Durchschnitt wurden Reformen in fünf Politikbereichen verfolgt. Die durchschnittliche Anzahl rein liberalisierender Reformen (2.9) lag dabei eindeutig über der Zahl von Reformen, die zwar auch häufig liberalisierten, dabei jedoch auch neue soziale Risiken abdeckten (1.9) ${ }^{4}$.

Diese Analyse von OECD-Daten zeigt, dass die Krise nicht genutzt wurde, um den Wohlfahrtsstaat zu modernisieren. Allerdings könnte es dennoch andere große Brüche mit der Vergangenheit im Bereich der Wirtschafts- und Sozialpolitiken gegeben haben, die nicht ins Blickfeld kommen, wenn man sich nur auf die OECD-Daten stützt.

Deshalb habe ich die Bertelsmann Datenbank über Reformpolitiken für die Jahre 2009 und 2011 ausgewertet. (http://www.sgi-network.org/, letzter Zugriff am 13. August 2012). Diese Datenbank enthält Experteneinschätzungen von verschiedenen Politikfeldern. Die Experten - in der Regel drei pro Land - wurden gebeten qualitative Informationen über verschiedenen Politiken zu liefern und diese dann bezüglich der Zielerreichung auf einer Rangskala einzustufen. Beispielsweise wurde gefragt, ob die Arbeitsmarktpolitik Arbeitslosigkeit wirksam in Angriff nimmt. Politiken wurden mit einem Kennwert zwischen 1 (vollkommener Misserfolg) und 10 (vollkommener Erfolg) versehen. Ich wertete die Informationen für eine breite Palette von Politiken aus ${ }^{5}$ und berechnete, ob sich die Ratings bedeutsam verändert haben. Als bedeutsam betrachtete ich jede Veränderung von zwei oder mehr Punkten -wobei die Skala zehn Punkte hatte. Die Ergebnisse sind substanziell identisch mit den Befunden der Analysen auf der Basis der OECD und FRDB Datenbanken: Im Allgemeinen gab es keinen bedeutsamen Bruch mit der Vergangenheit. Folgt man den Länderexperten, gab es nur in wenigen Ländern und in wenigen Politikfeldern große Veränderungen ${ }^{6}$.

Schließlich wertete ich eine vierte Datenquelle aus: das Political Data Yearbook des European Journal of Political Research. In diesem Jahrbuch berichten Länderexperten über große politische Ereignisse in den von mir untersuchten Ländern. Ich re-analysierte die

\footnotetext{
${ }^{4}$ Ich kodierte Politiken für Familien, vermehrte Bildung und Forschung und Steigerung der Beschäftigungsquote von älteren Arbeitnehmer (hauptsächlich durch Reduktion der Frühverrentung) als Politiken zur Deckung ,neuer sozialer Risiken'. Als rein liberalisierende Reformen kodierte ich Reformen, die den Wettbewerb steigern, soziale Sicherheit oder Steuern reduzieren, die kollektive und solidarische Lohnabkommen einschränken und den Immobilienmarkt von regulierenden Eingriffen und Subventionen befreien.

${ }^{5}$ Politiken in Bezug auf die Wirtschaft, den Arbeitsmarkt, die Unternehmen, das Gesundheitssystem, die soziale Inklusion, die Familien, die Renten, die Integration, die Forschung und Innovation und die Bildung.

${ }^{6}$ Bildungspolitik in Australien; Arbeitsmarktpolitik in Deutschland, Wirtschafts- und Rentenpolitik in Ungarn, Wirtschafts- Unternehmens-, Familien- und Bildungspolitik in Polen, Wirtschaftspolitik in Portugal und Unternehmenspolitik in der Schweiz.
} 
Berichte 2009, 2010 und $2011^{7}$ und fügte weitere Informationen aus Zeitschriftenarchive hinzu. Ich ging davon aus, dass die Länderexperten jede größere wirtschafts- und sozialpolitische Reform erwähnen würden. Falls die Krise Reformen auslöste, dann müssten sich hierfür Anzeichen in den Jahresberichten finden. Ich fand jedoch keine großen Reformpolitiken in den meisten Ländern. Die bisherigen Befunde eines Ausbleibens der breiten Politikinnovation werden also bestätigt.

Allerdings gab es häufig Veränderungen der nationalen politischen Systeme. Offenbar hat die Krise das Aufkommen fremdenfeindlicher oder rechtspopulistischer Einstellungen, Parteien oder Organisationen begünstigt. Zuweilen sahen sich auch Mitte-RechtsRegierungen oder sogar linke Regierungen gezwungen, Forderungen der rechtspopulistischen Parteien oder fremdenfeindlicher Wählerschaften aufzunehmen, wenn sie nicht massive Stimmenverluste riskieren wollten. Beispiele für solche Verschiebungen der nationalen Politik nach rechts finden sich in Australien, Dänemark, Finnland, Frankreich, Griechenland, den Niederlanden, Schweden und Ungarn (vergleiche auch Caramani et al. 2011: 870).

\section{Alternativlose Austerität}

Die Untersuchung der Policy-Reformen weist auf keine Innovationen hin. Aber es hat trotzdem beträchtlichen Wandel gegeben. Er betrifft den Umfang des Wohlfahrtsstaates. Regierungen versuchten einen weiteren Zuwachs zu verhindern und in vielen Fällen strebten sie an, ihn zurückzubauen. Beispielsweise wurde während der europäischen Staatsschuldenkrise in den südeuropäischen Ländern Austeritätsprogramme dramatischen Ausmaßes umgesetzt (Armingeon und Baccaro 2012). Die baltischen Länder, Irland und Großbritannien unter der liberal-konservativen Regierung Camerons beabsichtigten Ausgaben massiv zu senken. Selbst neu gewählte sozialdemokratische Regierungen, wie jene unter dem Sozialisten Hollande in Frankreich, dachten nicht daran, die Ausgaben insgesamt zu erhöhen. Allerdings variiert die Grösse der Sparprogramme beträchtlich zwischen den Ländern. Folgt man Manfred Schmidt, dann wäre zu erwarten, dass linke Regierungen das Sparprogramm geringer dimensionieren als konservativ-liberale Exekutiven (Schmidt 2010b; Schmidt 1996).

Eine zweite Erklärung der Varianz von Austeritätspolitik ist funktional. Das Ausmaß des Sparprogramms ist nur eine Folge der Intensität der vorhergehenden fiskalischen Probleme. Wenn Regierungen versuchen die Staatsfinanzen zu sanieren, ist die Rolle des

\footnotetext{
${ }^{7}$ Ich bin dem Herausgeber des Jahrbuches, Daniele Caramani, dankbar, dass er mir die vorliegenden Manuskripte für den im Dezember 2012 erscheinenden Band zugänglich machte. Nur sehr wenige Länderberichte fehlten oder waren Entwürfe, als ich die Unterlagen im Spätsommer 2012 auswertete.
} 
Parteienwettbewerbs nur sehr beschränkt - wenn er überhaupt eine Rolle spielt. Diese Erklärung wird vom Internationalen Währungsfonds (IMF 2010a) und von Schülern Schmidts vertreten (vgl. Wagschal und Wenzelburger 2008, 2012).

In meiner Analyse nutze ich Daten über die geplanten Konsolidierungen, also was die Regierungen im Vergleich zum Status Quo verändern möchten. Diese Daten stammen vom Internationalen Währungsfonds. Sie beruhen auf Regierungsangaben über deren Budgetentscheidungen und deren fiskalischen Konsequenzen. Wenn Parteipolitik eine Rolle spielen sollte, müsste man dies an diesen Daten sehen können. Sie stammen aus vier Ausgaben des ,Fiscal Monitor’ des Internationalen Währungsfonds (IMF 2010a, 2011 a,b, 2012). Jede dieser Publikationen enthält die historischen Daten über das Defizit - also das Defizit, das bisher verzeichnet wurde - und das geplante und angestrebte Defizit. Ich vergleiche in meiner Analyse die beabsichtigte Veränderung des um Konjunktureffekte bereinigten Defizits, das sogenannte, strukturelle Defizit' ('cyclically adjusted overall balance’). Dieses Maß zeigt den diskretionären Wandel an, d.h. den Wandel, den Regierungen beschlossen haben ${ }^{8}$. Für jedes Land habe ich insgesamt vier Messungen der fiskalischen Konsolidierung, d.h. je eine Messung von jeder Ausgabe des Fiscal Monitors. Jede dieser Messungen ist die Differenz zwischen dem Durchschnitt der geplanten Defizite im gegenwärtigen und den zwei zukünftigen Jahren und dem Durchschnitt der tatsächlichen Defizite in den vorhergehenden drei Jahren ${ }^{9}$. Ich replizierte dieses Vorgehen auch für historische und geplante Ausgaben gemessen in Prozent des Bruttoinlandprodukts. Es handelt sich dabei ebenfalls um geplante Veränderungen der Ausgaben. Diese Veränderungsraten wurden dann auf Vektoren von ökonomischen und politischen Variablen regressiert. Die ausgewählten ökonomischen Variablen sind Standardvariablen von ökonomischen Modellen zur Erklärung von Budgetkonsolidierungen: Das Niveau der Defizite (oder Ausgaben), gemittelt über die vorhergehenden drei Jahre, der Trend der historischen Defizite (oder Ausgaben), gemessen als Differenz des Durchschnitts der drei jüngsten Jahre minus dem Durchschnitt der drei zuvorliegenden Jahre, der Durchschnitt der Staatsschulden in Prozent des Bruttoinlandproduktes (Quelle: Fiscal Monitor) in den drei jüngsten Jahren, der

\footnotetext{
${ }^{8}$ Leider sind in meinen Quellen keine Daten zu den konjunkturbereinigten primären Defiziten zu finden. Primäre Defizite sind Defizite nach Abzug der Zinszahlungen auf Staatsschulden. Deshalb muss ich die konjunkturbereinigten Defizite vor Zinszahlungen benutzen. Für Malta, Luxembourg und Zypern fehlen Daten; diese Fälle müssen deshalb von der statistischen Analyse ausgeschlossen werden.

${ }^{9}$ Im Fall des Fiscal Monitor vom November 2010, beziehen sich die historischen Daten auf 2007, 2008 and 2009; im Fall des Fiscal Monitors vom April 2011 und vom September 2011 auf 2008, 2009, und 2010 und im Fall des Fiscal Monitor vom April 2012 auf2009, 2010, und 2011. Die geplanten Defizite beziehen sich auf 2010, 2011 und 2012 (Fiscal Monitor November 2010), 2011, 2012 und 2013 (Fiscal Monitor April und September 2011); und 2012, 2013, und 2014 (Fiscal Monitor 2012).
} 
Durchschnitt der nominalen Zinssätze auf Staatsanleihen mit zehnjähriger Laufzeit für die drei jüngsten Jahre (Quelle: OECD Economic Outlook, verschiedene Jahre http://Staatseinnahmen.oecd.org/eco/economicoutlook.htm, und

Eurostat http://epp.eurostat.ec.europa.eu/portal/page/portal/eurostat/home; fehlende Daten für Estland, Litauen (2008-11) und Rumänien), und das Niveau und den Trend der Arbeitslosigkeit (Quelle: OECD Labour Force Statistics http://stats.oecd.org/index.aspx?queryid=251 und Eurostat; Berechnungen analog zu den Berechnungen von Niveau und Trend im Falle von Defizite und Ausgaben). Die politischen Variablen sind der Sitzanteil linker Parteien im Kabinett, gemittelt über für die drei vorhergehenden Jahre. Eine alternative Operationalisierung beruht auf dem von Manfred Schmidt entwickelten Index der politischen Zusammensetzung der Regierung, der in der Forschung breit genutzt wird. Der Index geht von 1=Hegemonie rechter Parteien zu 5=Hegemonie linker Parteien. (Quelle mit Angaben zur Operationalisierung: Armingeon et al. 2011 und Aufdatierungen dieses Datensatzes).

Die Regressionsanalyse beruht auf robusten Driscoll und Kraay Standardfehlern ${ }^{10}$. Sie hat ferner fixe Zeiteffekte (d.h. Dummy Variablen für die jeweilige Ausgabe des Fiscal Monitors). Auch ohne fixe Zeiteffekte sind die substanziellen Ergebnisse identisch. Bei der Interpretation ist zu beachten, dass ich vom Saldo von Staatsausgaben und -einnahmen ausging; Defizite haben also ein negatives Vorzeichen. Zur Vermeidung von Missverständnissen habe ich die Defizit-Variable ,Saldo Staatseinnahmen/-ausgaben’ genannt. Ein positives Vorzeichen der Variablen zeigt einen Überschuss an, ein negatives Vorzeichen ein Defizit. Die Ergebnisse der Regressionsanalyse sind in Tabelle 1 abgetragen. Ich interpretiere Regressionskoeffizienten, wenn sie mindestens auf dem 0.1-Niveau für zweiseitige Test signifikant sind; dies bedeutet dass die bei gerichteten Hypothesen (und dies betrifft alle Variablen im Modell) auf dem 0.05-Niveau für einseitige Tests signifikant sind.

Tabelle 1: Angestrebte Veränderungen von Saldo und Ausgaben

\begin{tabular}{|c|c|c|c|c|c|c|}
\hline & $\begin{array}{c}\text { Modell 1 } \\
\text { Angestrebte } \\
\text { Veränderung } \\
\text { Saldo } \\
\end{array}$ & $\begin{array}{c}\text { Modell } 2 \\
\text { Angestrebte } \\
\text { Veränderung } \\
\text { Ausgaben } \\
\end{array}$ & $\begin{array}{c}\text { Modell 3 } \\
\text { Angestrebte } \\
\text { Veränderung } \\
\text { Saldo } \\
\end{array}$ & $\begin{array}{c}\text { Modell } 4 \\
\text { Angestrebte } \\
\text { Veränderung } \\
\text { Ausgaben } \\
\end{array}$ & $\begin{array}{c}\text { Modell 5 } \\
\text { Angestrebte } \\
\text { Veränderung } \\
\text { Saldo } \\
\end{array}$ & $\begin{array}{c}\text { Modell 6 } \\
\text { Angestrebte } \\
\text { Veränderung } \\
\text { Ausgaben } \\
\end{array}$ \\
\hline Niveau Saldo & $\begin{array}{l}-0.404 \\
(0.036)^{* * *}\end{array}$ & & $\begin{array}{l}-0.400 \\
(0.021)^{* * *}\end{array}$ & & $\begin{array}{l}-0.419 \\
(0.017)^{* * *}\end{array}$ & \\
\hline Trend Saldo & -0.089 & & -0.090 & & -0.088 & \\
\hline
\end{tabular}

\footnotetext{
10 'The error structure is assumed to be heteroskedastic, autocorrelated up to some lag, and possibly correlated between the groups (panels). Driscoll-Kraay standard errors are robust to very general forms of cross-sectional ("spatial") and temporal dependence when the time dimension becomes large.' Stata help xtscc.
} 


\begin{tabular}{|c|c|c|c|c|c|c|}
\hline & $(0.070)$ & & $(0.073)$ & & $(0.064)$ & \\
\hline Niveau Schulden & $\begin{array}{r}-0.002 \\
(0.002)\end{array}$ & $\begin{array}{l}-0.001 \\
(0.005)\end{array}$ & $\begin{array}{r}-0.001 \\
(0.001)\end{array}$ & $\begin{array}{r}0.004 \\
(0.005)\end{array}$ & $\begin{array}{l}-0.003 \\
(0.001)^{* *}\end{array}$ & $\begin{array}{r}0.005 \\
(0.005)\end{array}$ \\
\hline Zinssatz & $\begin{array}{l}0.408 \\
(0.042)^{* * *}\end{array}$ & $\begin{array}{l}-0.426 \\
(0.040)^{* * *}\end{array}$ & $\begin{array}{l}0.431 \\
(0.118)^{* *}\end{array}$ & $\begin{array}{l}-0.101 \\
(0.089)\end{array}$ & $\begin{array}{r}0.188 \\
(0.168)\end{array}$ & $\begin{array}{c}0.401 \\
(0.140)^{*}\end{array}$ \\
\hline $\begin{array}{l}\text { Niveau } \\
\text { Arbeitslosigkeit }\end{array}$ & $\begin{array}{r}0.095 \\
(0.046)\end{array}$ & $\begin{array}{l}-0.070 \\
(0.028)^{*}\end{array}$ & $\begin{array}{r}0.095 \\
(0.048)\end{array}$ & $\begin{array}{l}-0.108 \\
(0.020)^{* *}\end{array}$ & $\begin{array}{r}0.102 \\
(0.048)\end{array}$ & $\begin{array}{l}-0.120 \\
(0.024)^{* *}\end{array}$ \\
\hline $\begin{array}{l}\text { Trend } \\
\text { Arbeitslosigkeit }\end{array}$ & $\begin{array}{r}-0.018 \\
(0.080)\end{array}$ & $\begin{array}{l}-0.115 \\
(0.014)^{* * *}\end{array}$ & $\begin{array}{r}-0.017 \\
(0.080)\end{array}$ & $\begin{array}{l}-0.074 \\
(0.032)\end{array}$ & $\begin{array}{r}-0.032 \\
(0.081)\end{array}$ & $\begin{array}{r}-0.055 \\
(0.026)\end{array}$ \\
\hline Linksregierung & $\begin{array}{r}-0.003 \\
(0.002)\end{array}$ & $\begin{array}{r}-0.003 \\
(0.005)\end{array}$ & $\begin{array}{r}-0.000 \\
(0.013)\end{array}$ & $\begin{array}{c}0.031 \\
(0.010)^{*}\end{array}$ & & \\
\hline $\begin{array}{l}\text { Fiscal Monitor } \\
\text { April } 2011\end{array}$ & $\begin{array}{l}-0.638 \\
(0.107)^{* * *}\end{array}$ & $\begin{array}{l}0.816 \\
(0.119)^{* * *}\end{array}$ & $\begin{array}{l}-0.639 \\
(0.109)^{* * *}\end{array}$ & $\begin{array}{l}0.800 \\
(0.113)^{* * *}\end{array}$ & $\begin{array}{l}-0.638 \\
(0.107)^{* * *}\end{array}$ & $\begin{array}{l}0.753 \\
(0.099)^{* * *}\end{array}$ \\
\hline $\begin{array}{l}\text { Fiscal Monitor } \\
\text { Sept. } 2011\end{array}$ & $\begin{array}{l}-0.938 \\
(0.083)^{* * *}\end{array}$ & $\begin{array}{l}0.825 \\
(0.131)^{* * *}\end{array}$ & $\begin{array}{l}-0.940 \\
(0.084)^{* * *}\end{array}$ & $\begin{array}{l}0.808 \\
(0.125)^{* * *}\end{array}$ & $\begin{array}{l}-0.931 \\
(0.082)^{* * *}\end{array}$ & $\begin{array}{l}0.759 \\
(0.112)^{* * *}\end{array}$ \\
\hline Fiscal Monitor & -2.344 & 3.360 & -2.346 & 3.304 & -2.342 & 3.215 \\
\hline $\begin{array}{l}\text { April } 2012 \\
\text { Niveau Ausgaben }\end{array}$ & $(0.122)^{* * *}$ & $\begin{array}{l}(0.459)^{* * *} \\
-0.028 \\
(0.014)\end{array}$ & $(0.121)^{* * *}$ & $\begin{array}{l}(0.438)^{* * *} \\
-0.025 \\
(0.017)\end{array}$ & $(0.118)^{* * *}$ & $\begin{array}{l}(0.419)^{* * *} \\
-0.018 \\
(0.017)\end{array}$ \\
\hline Trend Ausgaben & & $\begin{array}{r}-0.149 \\
(0.160)\end{array}$ & & $\begin{array}{r}-0.188 \\
(0.156)\end{array}$ & & $\begin{array}{r}-0.209 \\
(0.165)\end{array}$ \\
\hline $\begin{array}{l}\text { Zinssatz * } \\
\text { Linksregierung }\end{array}$ & & & $\begin{array}{r}-0.000 \\
(0.003)\end{array}$ & $\begin{array}{l}-0.007 \\
(0.003)^{*}\end{array}$ & & \\
\hline $\begin{array}{l}\text { Regierungszusam- } \\
\text { mensetzung }\end{array}$ & & & & & $\begin{array}{l}-0.359 \\
(0.304)\end{array}$ & $\begin{array}{l}1.363 \\
(0.226)^{* * *}\end{array}$ \\
\hline $\begin{array}{l}\text { Zinssatz * Regierungs- } \\
\text { zusammensetzung }\end{array}$ & & & & & $\begin{array}{r}0.070 \\
(0.071)\end{array}$ & $\begin{array}{l}-0.298 \\
(0.060)^{* *}\end{array}$ \\
\hline Konstante & $\begin{array}{l}-2.193 \\
(0.526)^{* *}\end{array}$ & $\begin{array}{l}2.707 \\
(0.398)^{* * *}\end{array}$ & $\begin{array}{l}-2.297 \\
(0.677)^{* *}\end{array}$ & $\begin{array}{l}1.300 \\
(0.401)^{* *}\end{array}$ & $\begin{array}{r}-1.217 \\
(0.734)\end{array}$ & $\begin{array}{r}-1.229 \\
(0.575)\end{array}$ \\
\hline $\mathrm{R}^{2}$ & 0.76 & 0.60 & 0.76 & 0.61 & 0.76 & 0.62 \\
\hline $\mathrm{N}$ & 113 & 117 & 113 & 117 & 113 & 117 \\
\hline
\end{tabular}

Anmerkungen: ${ }^{* * *} p<.01,{ }^{* *} p<.05,{ }^{*} p<.10$. Standardfehler in Klammern.

Modelle 1 und 2 zeigen, dass die Zinssätze auf Staatsanleihen bei Weitem die konsistentesten und signifikantesten Prädiktoren der geplanten Änderungen von Defiziten und Staatsausgaben darstellen. Je höher die Zinssätze sind, die Regierungen auf ihre Schulden zahlen müssen, desto grösser fallen die angestrebten Defizit- und Ausgabenreduktionen aus. In diesen Modellen besitzt die parteipolitischen Zusammensetzungen der Regierungen keine bemerkenswerte Erklärungskraft. Die erste Schlussfolgerung lautet daher, dass im Allgemeinen die parteipolitische Zusammensetzung der Regierung keinen Unterschied für Konsolidierungsstrategien macht. In der ,großen Rezession', die 2007 beginnt, sind Regierungen zur Budgetkonsolidierung gezwungen, und die Konsolidierungsmaßnahmen sind eine Funktion der fiskalischen Probleme.

Aus der Perspektive Manfred Schmidts ist das nicht verwunderlich, hat er doch immer wieder die Bedeutung der Breite des Handlungskorridors für einen differenziellen Effekt von Parteien betont. Wo der Handlungskorridor verschwunden ist - wie beispielsweise in 
Südeuropa - ist auch kein Effekt politischer Parteien zu erwarten. Damit stellt sich aber die Frage, ob bei breiterem Handlungskorridor - wenn also die Zinssätze auf Staatsschulden gering sind - ein Parteieneffekt zu beobachten ist. Vielleicht gibt es einen Interaktionseffekt, so dass der Parteieneffekt desto grösser ist, je niedriger die Zinssätze sind. Ich habe deshalb einen Interaktionsterm von Zinssätze und parteipolitischer Zusammensetzung der Regierung in die Regressionsmodelle eingeführt.

Die Modelle 3 und 5 liefern jedoch keinen empirischen Beleg für einen derartigen Interaktionseffekt, wenn es um die Reduzierung des Defizits geht. Allerdings ist dieser Interaktionseffekt bei der Ausgabenplanung zu beobachten, wie Modelle 4 und 6 zeigen: Wenn die Zinssätze auf Staatsanleihen und damit die fiskalischen Probleme geringer sind, planen linke Regierungen weniger die Ausgaben zu reduzieren, als dies rechte Regierungen tun. Sie werden unter vorteilhaften fiskalischen Umständen stärker auf die Defizitreduktion durch Steuererhöhungen setzen. Dieser Zusammenhang existiert aber nur, wie die Berechnung entsprechender marginaler Effekte zeigt, wenn die Zinssätze niedrig sind. Die Zurückhaltung linker Regierungen verliert ihre statistische Signifikanz, sobald Zinssätze durchschnittlich oder überdurchschnittlich hoch sind.

Die substanzielle Schlussfolgerung dieser Analyse lautet folgendermaßen: Während der gegenwärtigen Krise ist das Ausmaß der beabsichtigten Defizitreduktionen eine Funktion der bestehenden fiskalischen Probleme. Je mehr ein Land in der fiskalischen Misere steckt, desto mehr beabsichtigt es zu sparen - völlig unabhängig von der parteipolitischen Zusammensetzung der Regierung. Bei günstigen fiskalischen Startpositionen vor der Krise können Regierungen jedoch zwischen verschiedenen Konsolidierungsstrategien wählen. Linke Regierungen bevorzugen eine Konsolidierung durch Steuererhöhungen, während rechte Regierungen auf Ausgabenkürzungen setzen. Sobald jedoch die fiskalischen Probleme grösser werden, verschwinden diese parteipolitischen Differenzen der Konsolidierungsstrategien.

\section{Was Märkte mögen}

Die Zinssätze auf Staatsanleihen sind ausgezeichnete Prädiktoren für das Ausmaß der Austeritätspolitik. Wie können wir die Varianz dieser Zinssätze erklären?

Darauf gibt es eine Standardantwort der Wirtschaftswissenschaftler. Die Zinssätze spiegeln die objektiven Risiken der Geldentwertung oder des Zahlungsausfalls wider. Märkte werden umso höhere Zinsen verlangen, je umfangreicher Schulden und Defizite sind und je geringer das Wirtschaftswachstum ist. Von diesen harten ökonomischen Grundzahlen leiten 
sich die Zinsforderungen ab. Dies ist demokratietheoretisch tröstend, weil Regierungen dann nur die Früchte ihres Erfolgs ernten oder die Strafen für ihr Versagens bekommen und die Marktentscheidung rational und unvoreingenommen ist. Zinssätze enthalten einen Basiszinssatz und eine Risikoprämie, die antizipierte Verluste aufgrund von Inflation oder Zahlungsausfall enthält ${ }^{11}$. In der wirtschaftswissenschaftlichen Literatur werden häufig diese theoretisch erwarteten Zusammenhänge empirisch berichtet, aber die Befunde sind erstaunlicherweise nicht immer sehr überzeugend und eindeutig, insbesondere wenn es sich um einen internationalen Vergleich von Zinssätzen handelt (Gale und Orszag 2003; Jaramillo und Weber 2012: 4). Ein offensichtliches Problem besteht in der Fokussierung der Marktteilnehmer auf die Zukunft und die schwache Stützung auf retrospektiver Datenauswertung (Laubach 2009). Die Vorhersage zukünftiger Entwicklungen der Inflationsoder Wachstumsrate oder der Zahlungsausfallswahrscheinlichkeit sind jedoch höchst fehleranfällig.

Rating Agenturen spielen eine zentrale Rolle in der Bewertung der Wirtschafts- und Regierungsleistungen der Vergangenheit und in der Abschätzung der Wahrscheinlichkeit eines Staatsbankrottes. Gärtner und Griesbach argumentierten in einer Analyse der europäischen Staatsschuldenkrise, diese Agenturen würden zwar auch objektive ökonomische Tatsachen berücksichtigen, aber sie seien darüber hinaus auch anfällig für Vorurteile, verzerrte Wahrnehmungen und einem wenig rationalen Reaktionsmuster (Gärtner und Griesbach 2012). Rating Agenturen sind weit weniger rational, informiert, unvoreingenommen als man annehmen möchte. Sie sind viel ideologischer und neigen zu Unter- oder Überreaktionen als dies ihr Anspruch auf angemessene Krisendiagnose erwarten lässt. Die Autoren zitieren G. Soros: 'I am not well qualified to criticize the theory of rational expectations and the efficient market hypothesis because as a market participant I considered them so unrealistic that I never bothered to study them' (Gärtner and Griesbach 2012: 13, Fußnote 16). Sie verweisen auch auf den Chef-Ökonomen des Internationalen Währungsfonds, Olivier Blanchard, der seine Lehren des Krisensjahres 2011 in vier Botschaften zusammenfasste, darunter ' the world economy is pregnant with multiple equilibria — selffulfilling outcomes of pessimism or optimism, with major macroeconomic implications; ... financial investors are schizophrenic about fiscal consolidation and growth. They react positively to news of fiscal consolidation, but then react negatively later, when consolidation leads to lower growth-which it often does; ...perception molds reality. Right or wrong, conceptual frames change with events. And once they have changed, there is no going back.

\footnotetext{
${ }^{11}$ In der empirischen Analyse verwende ich die Nominalzinssätze auf langfristige (10-jährige) Staatsanleihen als Indikatoren der Zinssätze auf Staatsschulden
} 
For example, nothing much happened in Italy over the summer. But, once Italy was perceived as at risk, this perception did not go away’ (Blanchard 2012). Und Bill Gross, der Gründer der global arbeitenden Investmentfirma 'Pimco' schrieb in der Financial Times: 'The dirty little secret that sovereign issuing nations need to remember most of all is that credit and maturity extension is based upon trust. After all, “credere” is a Latin word meaning just that.' http://www.ft.com/cms/s/0/36327c16-dd53-11e1-aa7b-00144feab49a.html, zuletzt zugegriffen am 6. August 2012).

Ganz offensichtlich resultiert die Entscheidung über die Höhe eines Zinssatzes auf Staatsschulden aus keiner einfachen Rechenübung, bei der makro-ökonomische Daten mit einem eindeutigen und gut begründeten Algorithmus verarbeitet werden. Wenn Wahrnehmung und Psychologie so wichtig sind, dann ist davon auszugehen, dass -jenseits der objektiven Regierungsleistungen - auch Merkmale des politischen Systems in die Entscheidung der Banker einfließen. Die zentrale Frage, die Rating Agenturen und Bankmanager zu beantworten haben, lautet ob die Regierung eines Landes fähig ist, solide Wirtschafts- und Fiskalpolitiken zu verfolgen. Deshalb könnten Konflikts-und Konsensprozesse, Institutionen und Staatstätigkeiten eine wichtige Rolle bei der Festlegung der Zinssätze spielen, selbst nachdem man die objektiven ökonomischen Daten berücksichtigt hat.

Die Literatur über die Wirkungen von politischen Kräfteverhältnissen und Institutionen, zu der Manfred Schmidt so viel beigetragen hat, erlaubt es einige Hypothesen abzuleiten. Dabei ist anzunehmen, dass die Bankmanager und Rating Agenturen auf vernünftige Weise ihr Eigeninteresse an minimierten Risiken und maximalen Erträgen verfolgen:

Investoren werden vermutlich linke und zentristische Parteien - wie die deutsche Christdemokratie - mit weniger Vertrauensvorschüssen versehen, als rechts-liberale Parteien, die sich eindeutig zu freien Märkten und minimaler Staatsintervention bekennen. Außer diesen ideologischen Aspekten könnten Marktakteure auch politische Institutionen in ihr Kalkül einbeziehen. In einer ökonomischen Krise könnten Regierungen besonders schnell, konsistent und entschlossen handeln, wenn die politische Macht in der nationalen Exekutiven konzentriert ist ${ }^{12}$. Deshalb sollten unitarische politische Systeme mit einem Mehrheitswahlrecht, das klare Mehrheiten und Ein-Parteien-Regierungen fördert, mehr Reputation bei Marktakteuren haben, als Bundesstaaten mit Verhältniswahlrecht und

\footnotetext{
${ }^{12}$ Das Argument, dass Machtkonzentration die Handlungsfähigkeit des politischen Systems auf externe Herausforderungen erhöht, liegt bekanntlich axiomatisch den Arbeiten von Fritz W. Scharpf zugrunde; siehe den Beitrag von Scharpf in diesem Band oder beispielsweiseScharpf 1988.
} 
Koalitionsregierungen. Andererseits - und darauf macht Manfred Schmidt in seiner Abwägung von Vorzügen und Nachteilen von Westminster- und Konsensdemokratie in seinem Buch über Demokratietheorien aufmerksam (Schmidt 2010a) - neigen Wettbewerbsdemokratien zu Zick-Zack-Politiken, die der Wahlkalender diktiert, zu mehr erratischen Politiken und ineffizienter Politikimplementation. Systeme mit vielen Vetopunkten und dem Zwang zur Konzertierung sind vorhersagbarer und produzieren besser durchdachte und dauerhafte Politiken, die aufgrund des breiten Konsens in der Phase der Politikformulierung besser implementiert werden. Verhandlungsdemokratien mögen schlechter darin sein, schnell zu reagieren, aber dies schützt sie auch vor schnell gemachten Fehlern, da jede Politikänderung gut begründet und verteidigt werden muss. Dies ist bekanntlich auch das zentrale Argument, weshalb Verhandlungsdemokratien Politikergebnisse haben, die nicht schlechter und gelegentlich sogar besser sind, als die Politikergebnisse in Westminster-Demokratien (Lijphart 1999; Armingeon 2002).

Marktakteure könnten auch politische Stabilität als wesentliches Bewertungskriterium heranziehen. Falls es häufig einen Regierungswechsel gibt oder wenn die Arbeitsbeziehungen durch viele Streiks und Aussperrungen geprägt sind, dann könnten Zweifel entstehen, ob dieses instabile politische System erfolgreich Krisen bekämpfen kann. Vielleicht vergrößern konflikthafte Arbeitsbeziehungen und chaotische Prozesse der Regierungsbildung sogar das Ausmaß der Wirtschaftsprobleme. Bekanntlich hat Peter Katzenstein anhand der europäischen Kleinstaaten gezeigt, dass politische und gesellschaftliche Stabilität eine Ursache des wirtschaftlichen Erfolges angesichts globaler Herausforderungen ist (Katzenstein 1985, 2003).

Schließlich könnten auch Umfang und die Entwicklung des Wohlfahrtsstaates ein wichtiger Bewertungsfaktor von Marktakteure sein. Der Umfang des öffentlichen Sektors den man anhand der Staatseinnahmen in \% des Bruttoinlandsproduktes messen könnte - und die Größe des Wohlfahrtsstaates, welche man am Umfang der sozialpolitischen Transfers (in \% des BIP) ablesen könnte, könnten ganz unterschiedliche Signale an die Märkte senden. Einerseits haben Länder mit einem großen öffentlichen Sektor und Wohlfahrtsstaat eine Reputation als stabile und erfolgreiche Volkswirtschaften - man denke nur an Schweden oder Deutschland. Andererseits passen umfangreiche Wohlfahrtsstaaten wahrscheinlich nicht in die Weltsichten von vielen Bankern und Ökonomen in Rating-Agenturen, die davon ausgehen, dass Märkte effizienter und erfolgreicher sind, als alle alternativen Regelungsformen. Während Märkte sich vielleicht an einen etablierten Staatssektor gewöhnt haben, könnten sie vor allem ein rasches Wachstum der Staatseinnahmen und der Sozialausgaben als dramatisches Signal einer sich verschlechternden Wirtschaftslage interpretieren. 
Ich überprüfte diese Hypothesen auf der Basis eines ländervergleichenden Datensatzes für den Zeitraum zwischen 1960 und 2011 und für die 27 EU Länder sowie der anderen etablierten Demokratien außerhalb der EU. Daten wurde nur für konsolidierte Demokratien analysiert. Die Zahlenreihen für Malta und Zypern reichen nicht vor 1990 zurück. Es gibt in dem Datensatz zahlreiche fehlende Werte, insbesondere für die 1960er und 1970er Jahre.

Die abhängige Variable ist der standardisierte nominale Zinssatz für langfristige Staatsanleihen (d.h. Staatsanleihen mit einer 10-jährigen Laufzeit). Die Standardisierung ist notwendig, um zeitspezifische Effekte zu eliminieren, die alle Länder gleichermaßen betroffen haben. Da es sehr viele fehlende Werte gibt, würde die Subtraktion des jahrespezifischen Mittelwertes wenig helfen, da diese Zeitreihen vielfache Brüche aufweisen würden. Deshalb folgte ich der üblichen Praxis in der wirtschaftswissenschaftlichen Forschung (beispielsweise Gärtner und Griesbach 2012) und standardisierte die Zinssätze mit dem Zinssatz auf deutsche Staatsanleihen, für den wir für alle Jahre zwischen 1960 und 2011 Angaben haben. Folglich ist die abhängige Variable in den Regressionsanalysen die Differenz zwischen dem jeweiligen nationalen und dem deutschen Zinssatz auf langfristige Staatsanleihen. Bei der Auswertung solcher internationaler und gepoolter Zeitreihen wird standardmäßig die abhängige Variable auf der rechten Gleichungsseite um ein Jahr zeitverzögert aufgenommen, um eine serielle Korrelation des Fehlerterms zu vermeiden (Beck und Katz 2011). Da jedoch die zeitverzögerte Variable auch mit dem Fehlerterm korreliert ist, führt dies zu einem verzerrten Schätzer (Baltagi 2010: 147-148). Ich habe diese Probleme reduziert, indem ich die abhängige Variable mit einer weiteren Zeitverzögerung um zwei Jahre eingeführt habe und Driscoll-Kraay Standardfehler für alle Schätzungen berechnet habe Zusätzlich habe ich die Befunde noch auf Robustheit geprüft, indem ich fixe Zeiteffekte in das Modell einführte und Modelle schätzte, bei der die abhängige Variable differenziert wurde, d.h. es wurde die Differenz zwischen der Differenz von nationalem und deutschem Zinssatz zwischen den Jahren t und t-1 berechnet. Die Daten stammen aus meinen öffentlich zugänglichen Datensätzen und deren Aufdatierungen für den Zweck dieser Analyse (Armingeon et al. 2011; Armingeon et al. 2012). Operationale Definitionen und Quellen sind in den entsprechenden Codebüchern dokumentiert ${ }^{13}$. Einige der theoretisch interessanten Variablen hatten Koeffizienten, die häufig das 0.10 Signifikanzniveau knapp verfehlten. Deshalb habe ich auch Koeffizienten auf dem 0.15 Signifikanzniveau markiert. Da die meisten Hypothesen gerichtet sind, müssen nur einseitige Tests vorgenommen werden und ein

\footnotetext{
${ }^{13}$ http://www.ipw.unibe.ch/content/team/klaus_armingeon/comparative_political_data_sets/index_ger.html
} 
Signifikanzniveau von 0.15 für zweiseitige Tests entspricht einem Signifikanzniveau von 0.075 - einer Irrtumswahrscheinlichkeit von 7.5\% - für einseitige Tests.

Tabelle 2: Regression Zinssatz (LDV-Modell)

\begin{tabular}{|c|c|c|c|c|c|}
\hline & $\begin{array}{c}\text { Zinssatz } \\
\text { Modell } 1 \\
\end{array}$ & $\begin{array}{c}\text { Zinssatz } \\
\text { Modell } 2 \\
\end{array}$ & $\begin{array}{r}\text { Zinssatz } \\
\text { Modell } 3 \\
\end{array}$ & $\begin{array}{c}\text { Zinssatz } \\
\text { Modell } 4 \\
\end{array}$ & $\begin{array}{r}\text { Zinssatz } \\
\text { Modell } 5\end{array}$ \\
\hline Zinssatz_Lag1 & $\begin{array}{l}0.929 \\
(0.108)^{* * * *}\end{array}$ & $\begin{array}{l}0.883 \\
(0.098) * * * *\end{array}$ & $\begin{array}{l}0.958 \\
(0.103) * * * *\end{array}$ & $\begin{array}{l}0.883 \\
(0.096) * * * *\end{array}$ & $\begin{array}{l}0.891 \\
(0.106) * * * *\end{array}$ \\
\hline Zinssatz_Lag2 & $\begin{array}{l}-0.089 \\
(0.111)\end{array}$ & $\begin{array}{l}-0.076 \\
(0.099)\end{array}$ & $\begin{array}{l}-0.139 \\
(0.092)^{*}\end{array}$ & $\begin{array}{r}-0.072 \\
(0.100)\end{array}$ & $\begin{array}{r}-0.072 \\
(0.110)\end{array}$ \\
\hline Schulden & $\begin{array}{l}-0.001 \\
(0.002)\end{array}$ & $\begin{array}{l}-0.001 \\
(0.002)\end{array}$ & $\begin{array}{r}-0.002 \\
(0.002)\end{array}$ & $\begin{array}{l}-0.001 \\
(0.002)\end{array}$ & $\begin{array}{l}-0.001 \\
(0.002)\end{array}$ \\
\hline Schulden_Trend & $\begin{array}{r}-0.007 \\
(0.007)\end{array}$ & $\begin{array}{r}-0.004 \\
(0.005)\end{array}$ & $\begin{array}{r}-0.003 \\
(0.004)\end{array}$ & $\begin{array}{r}-0.004 \\
(0.005)\end{array}$ & $\begin{array}{l}-0.010 \\
(0.007)^{*}\end{array}$ \\
\hline Saldo & $\begin{array}{l}-0.041 \\
(0.025)^{*}\end{array}$ & $\begin{array}{l}-0.046 \\
(0.022)^{* * *}\end{array}$ & $\begin{array}{l}-0.036 \\
(0.018)^{* * *}\end{array}$ & $\begin{array}{l}-0.044 \\
(0.021)^{* * *}\end{array}$ & $\begin{array}{l}-0.067 \\
(0.026)^{* * *}\end{array}$ \\
\hline Wirtschaftswachstum & $\begin{array}{l}0.016 \\
(0.033)\end{array}$ & $\begin{array}{l}0.032 \\
(0.028)\end{array}$ & $\begin{array}{r}0.029 \\
(0.026)\end{array}$ & $\begin{array}{r}0.032 \\
(0.027)\end{array}$ & $\begin{array}{r}0.019 \\
(0.036)\end{array}$ \\
\hline Regierungswechsel & & $\begin{array}{l}0.066 \\
(0.039)^{*}\end{array}$ & $\begin{array}{l}0.076 \\
(0.047)^{*}\end{array}$ & $\begin{array}{l}0.072 \\
(0.046)^{*}\end{array}$ & $\begin{array}{l}0.103 \\
(0.060)^{* *}\end{array}$ \\
\hline Gov_New & & $\begin{array}{r}0.007 \\
(0.097)\end{array}$ & $\begin{array}{r}-0.079 \\
(0.063)\end{array}$ & & \\
\hline Föderalismus & & $\begin{array}{r}-0.036 \\
(0.105)\end{array}$ & $\begin{array}{r}-0.042 \\
(0.092)\end{array}$ & & \\
\hline Stabile Koalition & & $\begin{array}{l}-0.017 \\
(0.092)\end{array}$ & $\begin{array}{r}-0.015 \\
(0.089)\end{array}$ & & \\
\hline $\begin{array}{l}\text { Wohlfahrtsstaat } \\
\text { (Ausmaß) }\end{array}$ & & $\begin{array}{l}-0.033 \\
(0.010)^{* * * *}\end{array}$ & $\begin{array}{l}-0.020 \\
(0.010)^{* * *}\end{array}$ & $\begin{array}{l}-0.033 \\
(0.009) * * * *\end{array}$ & $\begin{array}{l}-0.027 \\
(0.011)^{* * *}\end{array}$ \\
\hline Staatseinnahmen & & $\begin{array}{l}0.025 \\
(0.009)^{* * * *}\end{array}$ & $\begin{array}{l}0.022 \\
(0.007)^{* * * *}\end{array}$ & $\begin{array}{l}0.025 \\
(0.008) * * * *\end{array}$ & $\begin{array}{l}0.026 \\
(0.007)^{* * * *}\end{array}$ \\
\hline Gov_NewGap & & $\begin{array}{l}-0.014 \\
(0.050)\end{array}$ & $\begin{array}{r}0.047 \\
(0.043)\end{array}$ & & \\
\hline$\Delta$ Staatssektor & & $\begin{array}{l}0.049 \\
(0.027)^{* *}\end{array}$ & $\begin{array}{l}0.068 \\
(0.029)^{* * *}\end{array}$ & $\begin{array}{l}0.048 \\
(0.027)^{* *}\end{array}$ & $\begin{array}{l}0.086 \\
(0.029) * * * *\end{array}$ \\
\hline$\Delta$ Welfare & & $\begin{array}{c}0.231 \\
(0.144)^{*}\end{array}$ & $\begin{array}{l}0.105 \\
(0.074)\end{array}$ & $\begin{array}{l}0.232 \\
(0.146)^{*}\end{array}$ & \\
\hline Rechtsregierung & & $\begin{array}{r}-0.001 \\
(0.001)\end{array}$ & $\begin{array}{l}-0.001 \\
(0.001)\end{array}$ & $\begin{array}{c}-0.001 \\
(0.001)^{*}\end{array}$ & \\
\hline Streik_Lag1 & & & $\begin{array}{l}0.000 \\
(0.000)^{* * * *}\end{array}$ & & \\
\hline Konstante & $\begin{array}{r}0.175 \\
(0.334)\end{array}$ & $\begin{array}{r}-0.560 \\
(0.570)\end{array}$ & $\begin{array}{r}-0.589 \\
(0.493)\end{array}$ & $\begin{array}{r}-0.605 \\
(0.483)\end{array}$ & $\begin{array}{r}-0.585 \\
(0.548)\end{array}$ \\
\hline$\overline{\mathrm{R}^{2}}$ & 0.80 & 0.82 & 0.86 & 0.82 & 0.81 \\
\hline $\mathrm{N}$ & 792 & 760 & 677 & 761 & 761 \\
\hline
\end{tabular}

Anmerkungen: ${ }^{* * * *} p<.01 ;{ }^{* * *} p<.05 ;{ }^{* *} p<.10 ;{ }^{*} p<.15$. Standardfehler in Klammern.

In einem ersten Schritt regressierte ich den Zinssatz auf seinen, um ein und zwei Jahre zeitverzögerten Wert zusammen mit Standardvariablen der ökonomischen Forschung: dem 
staatlichen Schuldenstand (in \% des BIP), dem langfristigen Trend der Schuldenentwicklung, gemessen als dem durchschnittlichen Schuldenstand des aktuellen Jahres und der zwei vorhergehenden Jahre (t, t-1, t-2) abzüglich des durchschnittlichen Schuldenstandes in den drei davorliegenden Jahren (t-3,t-4,t-5); dem staatlichen Primärsaldo - bekanntlich zeigt ein positiver Wert einen Überschuss und ein negativer Wert ein Defizit an - sowie der nominalen Wachstumsrate. Dem Modell 1 der Tabelle 2 ist zu entnehmen, dass der um ein Jahr zeitverzögerter Zinssatz große prädiktive Kraft hat und der Koeffizient der Saldo-Variable erfüllt das 0.15 Signifikanzkriterium und ist sogar in den Modellen 2 bis 5 mit einer noch geringeren Irrtumswahrscheinlichkeit behaftet. Wie erwartet reagieren Märkte auf objektive historische Wirtschaftsdaten. Die Größe des Staatsdefizits ist ein weiterer und wichtiger fiskalischer Kennwert. Ich diskutiere diese Befunde aus Modell 1 nicht weiter, da der einzige Zweck dieser Berechnung darin bestand, ein Modell mit angemessenen ökonomischen Kontrollvariablen zu entwickeln. Die fehlenden starken Korrelationen zwischen Defiziten, Schuldenstand und Wirtschaftswachstum sind angesichts der uneinheitlichen Forschungsbefunde in der ökonomischen Literatur keine große Überraschung. Der nichtsignifikanten Koeffizient für den Schuldenstand kann auf langfristige Entwicklungen zurückgehen und die Tatsache, dass es einige hochverschuldete Länder - wie beispielsweise Japan - gibt, die dennoch einen niedrigen Zinssatz aufweisen.

Beim Modell 2 wurden politisch-institutionelle Variablen integriert. Eine DummyVariable für Föderalismus (1 für Australien, Belgien, Deutschland, Kanada, Österreich, Spanien, die Schweiz, die USA und ab 2001 Italien), die Zahl der Regierungswechsel pro Jahr (Gov_Change) ${ }^{14}$, eine Dummy-Variable, ob sich die ideologische Ausrichtung der neunen Regierung von jener der vorhergehenden unterscheidet (Gov_New), eine Dummy-Variable für eine strukturell stabile Regierungskoalition (1 für eine Koalition, die gerade nur soviel Parteien einschließt um eine Parlamentsmehrheit zu bilden oder eine übergroße (,surplus') Koalition, 0 für Ein-Parteien-Regierungen, Minderheiten- oder Interims/technische Regierungen). Der Umfang des Wohlfahrtsstaats wurde mit den Sozialtransfers in \% des BIP gemessen. Die Staatseinnahmen (in \% des BIP) zeigen den Umfang des öffentlichen Sektors an. Die ideologische Distanz zwischen der alten und der neuen Regierung (Gov_New_Gap) basiert auf dem Schmidt-Index der parteipolitischen Zusammensetzung der Regierung ('govparty' im Comparative Political Data Set). Die Wachstumsrate des öffentlichen Sektors ist die Differenz zwischen den Staatseinnahmen (in \% des BIP) im Jahr t und t-1. Das

\footnotetext{
${ }^{14}$ In einer alternativen Spezifikation kodierte ich dies auch als eine binäre Variable - 0 kein Wechsel, 1 mindestens ein Wechsel. Die Ergebnisse sind substantiell identisch, da mehr als ein Regierungswechsel pro Jahr nur sehr selten erfolgt.
} 
Wachstum des Wohlfahrtsstaates wird als Differenz der Sozialausgaben im Jahr t und t-1 gemessen. Ferner werden die Sitzanteile von linken, christdemokratisch/zentristischen und rechten Parteien in das Modell eingeführt. Da nur rechte Parteien einen signifikanten Regressionskoeffizienten hatten, werden nur Modelle mit rechten Parteien berichtet ${ }^{15}$. Leider fehlen Daten zu Streiks und Aussperrungen (Streiks und Aussperrungen im Jahr t-1 gemessen als verlorene Arbeitstage aufgrund von Streiks und Aussperrungen pro 1'000 Arbeitnehmer) für viele Länder. Deshalb wurde Modell 3 geschätzt, das diese Streikdaten verwendet. Um Informationsverluste aufgrund vieler fehlenden Daten zu vermeiden, wurden die anderen Modelle ohne die Arbeitskampfvariable geschätzt.

In einem weiteren Schritt eliminierte ich schrittweise rückwärts die politischinstitutionellen Variablen mit ganz geringer Signifikanz. Die ökonomischen Kontrollvariablen verblieben jedoch im Modell, unabhängig von deren Signifikanz. Model 4 besteht nur aus jenen Variablen des ursprünglichen Modells 2, die das niedrige Niveau einer Irrtumswahrscheinlichkeit von kleiner 20\% (oder bei einseitigen Fragestellung von weniger als $10 \%$ ) erreichten. In einem weiteren Schritt wurden jene politisch-institutionellen Variablen des Modells 4 ausgeschlossen, die nicht das 10\% Signifikanzniveau (zweiseitig) erreichten. Modell 5 enthält nur jene Variablen, die signifikant blieben. Dieses Modell wurde auf Robustheit geprüft. Es wurden fixe Zeiteffekte (Jahres-Dummies) eingeführt und in einer anderen Spezifikation wurden alle Modelle mit einer differenzierten abhängigen Variable (erste Differenzen der standardisierten Zinssätze) geschätzt. Die Logik der schrittweisen Modellbildung war analog zur Logik die die Schätzungen der Tabelle 2 leitete. Substantiell sind die Resultate identisch (siehe Tabellen 3 und 4 im Anhang).

Was zeigen einem diese statistischen Analysen? Ich konzentriere mich auf Modell 4 das alle Variablen enthält die mindestens auf dem einseitigen 10\% Niveau signifikant sind sowie auf das Modell 3, das als einziges die Arbeitskampfvariable enthält. Der erste wichtige Befund betrifft jene Faktoren, die für Märkte unwichtig sind: Die Machtkonzentration ist nicht wichtig. Weder bevorzugen Märkte Staaten mit konzentrierter politischer Macht, noch haben sie eine Vorliebe für Verhandlungsdemokratien mit vielen Vetopunkten. Weder der Vorteil von Wettbewerbsdemokratien - die Fähigkeit zur schnellen und konsistenten Reaktion - wird von Märkten geschätzt noch schlägt sich der Vorteil der Verhandlungsdemokratien -

\footnotetext{
${ }^{15}$ Mit guten Gründen kann man - und nicht zuletzt auf der Basis der Arbeiten von Manfred Schmidt vermuten, dass Märkte keine großen Bewertungsunterschiede zwischen sozialdemokratischen und christdemokratischen Regierungen machen: beide Parteifamilien sind Triebkräfte des Wohlfahrtsstaats, und eines großen öffentlichen Sektors, während nur rechte Parteien - und dies schließt die wirtschaftsliberalen Parteien ein - sich der Ausbreitung des Wohlfahrtsstaates widersetzt haben und für einen schlanken Staat plädierten, der möglichst wenig in die Wirtschaft eingreift.
} 
die hohe Vorhersagbarkeit, der Schutz vor Überreaktionen und geringere Bedeutung des Wahlkalenders für die Politikentwicklung - statistisch überzeugend nieder ${ }^{16}$.

Im Gegensatz dazu registrieren Märkte politische Instabilität (Regierungswechsel). Dabei wird weniger die Richtung des Wandels - von rechts nach links oder umgekehrt -, sondern vielmehr der Wandel selbst mit Besorgnis verzeichnet. Das Arrangement der Daten als Jahresdaten gestattet keine präzise Bestimmung der Kausalität: Gab es zunächst in einem Jahr einen Regierungswechsel, der die Märkte beunruhigte oder war die politische Situation chaotisch, was dann unabhängig voneinander zu einem Anstieg der Zinssätze und zu einem Regierungswechsel führte? Eine statistische Analyse, bei der die Zinssatzveränderungen auf die Regierungswechselvariablen im vorhergehenden Jahr zurückgeführt wurden - dass also der Regierungswechsel dem Anstieg des Zinssatzes vorausging und damit als Ursache gelten könnte -, erbrachten keine eindeutigen Befunde. Ebenso unklar waren die Ergebnisse der Regressionsanalysen, bei denen die Zinssatzveränderungen zeitlich dem Regierungswechsel vorausgingen. In diesem Fall könnte man vermuten, eine chaotische innenpolitische Situation führe Märkte dazu, den Zinssatz zu erhöhen, was dann auch mit einem politischen Wechsel im Folgejahr einherging. Märkte schätzen auch den Arbeitsfrieden. Wenn ein Land ein System der Arbeitsbeziehungen hat, in dem Arbeitskämpfe nicht die ultima ratio der Konfliktregulierung ist (wie in Deutschland, Österreich oder der Schweiz), dann hat dies positive Folgen für den Zinssatz. Obwohl Märkte einen großen öffentlichen Sektor nicht begrüßen, sind sie gegenüber dem Ausmaß des Wohlfahrtsstaates indifferent - wie dies die Modellschätzungen nahelegen. Eine mögliche Erklärung bezieht sich darauf, dass einige wirtschaftlich sehr erfolgreiche und stabile Länder wie die nordischen Länder, die Niederlande oder Deutschland auch einen großen Wohlfahrtsstaat haben, während dieser in den wirtschaftlich weniger erfolgreichen Nationen in Südeuropa klein ist. Wohlfahrtsstaatliche Sicherungssysteme stellen eine Produktivkraft moderner Gesellschaften dar und die Märkte anerkennen dies offensichtlich.

Zusätzlich zur politischen Stabilität, dem Arbeitsfrieden und einer erfolgreichen Wirtschaft, gibt es drei Faktoren, die unsere Modelle enthalten und die Märkte mögen: Sie möchten einen schlanken Staat. Sie honorieren einen Rückbau eines großen öffentlichen Sektors und des Wohlfahrtsstaates. Und schließlich haben sie eine leichte Präferenz für rechtsliberale Regierungen. Wenn sich das Volk in einem Lande in demokratischen Wahlen für eine linke oder zentristische (in der Regel: christdemokratische) Regierung entscheidet und wenn das Parlament sich für eine Beibehaltung oder gar Vergrößerung des öffentlichen

\footnotetext{
${ }^{16}$ Entsprechend zeigten sich in alternativen Spezifikationen keine systematischen Effekte des Wahlrechtes. Für Märkte ist weder ist das Mehrheitswahlrecht noch das Verhältniswahlrecht eindeutig überlegen.
} 
Sektors und der wohlfahrtsstaatlichen Transferzahlungen entscheidet, dann muss dafür ein Preis im Sinne höherer Zinssätze gezahlt werden.

Freilich macht ein Vergleich der verschiedenen Modelle der Tabelle 2 klar, dass die Erklärungskraft von politisch-institutionellen Variablen - zusätzlich zu den ökonomischen Variablen - durchaus begrenzt ist. Das Gleiche gilt auch für die Größe und Signifikanz der Koeffizienten. Ein Wechsel von einer rechten zu einer linken Regierung ist mit einem Anstieg des Zinssatzes um 0.13 Prozentpunkte verbunden und ein Regierungswechsel in einem Jahr führt zu einem Zinsanstieg um 0.1 Prozentpunkte in Modell 5. Allerdings muss auch erwähnt werden, dass nicht nur die politisch-institutionellen Variablen, sondern auch die ökonomischen Faktoren von begrenztem Erklärungswert für die Entwicklung der Zinssätze sind. Die einzige Ausnahme bildet die Höhe des vorjährigen Zinssatzes. Aber dies ist auch ein theoretisch interessanter Befund: Zinssätze sind offenbar ,sticky’ und Märkte ändern nicht schnell ihre Meinungen aufgrund veränderter ökonomischer Performanzindikatoren, wenn einmal die Meinung gemacht ist. Dies ist eine der wichtigen Lektionen, die O. Blanchard lehrt -und dies weckt erhebliche Zweifel über die Fähigkeit von Märkten, angemessen auf ökonomische Veränderungen zu reagieren.

Der zentrale Befund-Märkte mögen keinen großen und wachsenden Staatssektor und keinen wachsenden Wohlfahrtsstaat, sie honorieren politische Stabilität und sie bevorzugen rechte Regierungen - erweist sich in unterschiedlichen Modellspezifikationen als relativ robust (vgl. auch die Übersicht in Tabelle 5 im Anhang, die zeigt welche politischinstitutionellen Variablen bei welchem gewählten Signifikanzniveau statisch von Null unterschiedliche Regressionskoeffizienten haben).

\section{Schlussfolgerung}

Im Gegensatz zu den großen weltwirtschaftlichen Krisen der vergangenen 150 Jahre führte die große Rezession seit 2007 nicht zu massiven Reformen der Sozial- und Wirtschaftspolitik in den ökonomisch hochentwickelten Demokratien. Reformen bezogen sich lediglich auf die Konsolidierung der öffentlichen Haushalte. Die parteipolitische Zusammensetzung der Regierung beeinflusste in der Regel diese Austeritätspolitik nicht - allerdings mit der bemerkenswerten Ausnahme, dass unter vergleichsweise günstigen fiskalischen Bedingungen linke Regierungen stärker Steuererhöhungen zu Konsolidierung nutzten, während rechte Regierungen eher auf Ausgabenkürzungen setzten. Ein guter Prädiktor für das angestrebte Ausmaß der fiskalischen Konsolidierung ist der Zinssatz auf die öffentlichen Schulden. 
Dieser Zinssatz ist jedoch nicht eine ausschließliche Funktion ökonomischer Variablen. Der Zinssatz spiegelt auch die ideologischen Orientierungen der Banker und Rating Agenturen wider, welche politische Stabilität und rechte Regierungen präferieren.

Diese Ergebnisse führen mindestens zu zwei Folgefragen: (1) Gibt es demokratietheoretisch begründete Bedenken, wenn Märkte die Bürger einer Nation mit hohen Zinssätzen bestrafen, falls sie sich für eine linke oder Zentrumsregierung oder für einen chaotischen politischen Prozess oder für einen großen und wachsenden Staatssektor aussprechen? (2) Warum spielten in dieser Krise die parteipolitische und gesellschaftliche Kräfteverteilungen sowie Koalitionen eine so viel kleinere Rolle als in historischen Krisen vergleichbaren Ausmaßes?

Die erste Frage ist selbstverständlich eine normative Frage. Geht man von der Vorstellung aus, dass Märkte vollkommen rational und effizient sind und die objektiven Kosten politischer Entscheidungen in die Zinssätze einpreisen, dann erübrigt sich jede normative Kritik. Agieren Märkte jedoch nur begrenzt rational, dann besitzen die empirischen Ergebnisse erhebliche Implikationen. In diesem Fall wird der demokratische Entscheidungsspielraum durch die Ideologien und Stereotypen einiger weniger Banker und Rating Agenturen eingeschränkt. Es gibt überzeugende empirische Belege dafür, dass linke und Zentrumsregierungen nicht weniger wachstumsfreundlich sind als rechte Regierungen und schlanke Wohlfahrtsstaaten. Die zeigte unter anderem der Schmidt-Schüler Herbert Obinger (Obinger 2004; siehe auch Hall und Soskice 2001; Pontusson 2005; Mares 2010). Wenn Marktakteure ihre wirtschaftliche Macht nutzen, um einige Regierungen zu schärferen Austeritätspolitiken zu zwingen als anderen - unter sonst gleichen ökonomischen Bedingungen - dann haben sie eine doppelte Stimme: Als Staatsbürger und - viel, viel gewichtiger - als Wirtschaftseliten, deren Ideologien für den politischen Prozess erheblich mehr wiegen als jene der anderen Bürger. Das verletzt erneut den Grundsatz der politischen Gleichheit in Demokratien. Eine zweite Schlussfolgerung lässt sich ziehen: In der politischen Debatte wird immer wieder das Argument vorgebracht, Märkte seien effizienter und rationaler als politische Systeme und auch aus diesem Grund verliere der Staatseingriff in die Ökonomie seine Berechtigung. Dieses Argument wird entwertet, wenn Marktakteure ideologisch und nicht ökonomisch rational handeln. Für politische Akteure verringert sich damit die Notwendigkeit, Märkte und Marktprozesse politisch zu respektieren.

Die zweite Frage ist weit weniger normativ. Warum spielte die Parteipolitik und die gesellschaftlichen Kräfteverteilungen in dieser Krise im Gegensatz zu früheren Krisen kaum eine Rolle? Weshalb hilft uns beispielsweise die von Manfred Schmidt maßgeblich geprägte 
Parteiendifferenzthese in diesem Fall so viel weniger als in früheren wirtschaftlichen Notzeiten? Ich habe fünf Argumente, die im Grundsatz darauf hinauslaufen, dass der politische Kontext dieser Krise sich bedeutend von jenem in der Vergangenheit unterscheidet (siehe hierzu auch Hall 2013).

(1) Keine neuen Koalitionen. Peter Gourevitch erklärte große Reformen während Krisen mit dem Aufkommen neuer gesellschaftlicher und politischer Koalitionen. Krisen destabilisierten bisherige Koalitionen zwischen sozialen Gruppen und ihren politischen Vertretern und führten zu neuen Koalitionen, die neue Politiken verfolgen (Gourevitch 1986). Seit 2007 vollzogen sich zwar zahlreiche Regierungswechsel, aber bislang wurden noch keine grundsätzlich neuen politischen Konstellationen sichtbar. Vielmehr machen Wählerinnen und Wähler das, was erfolglose Fußballvereine tun: Sie feuern den Trainer - selbst wenn die Spieler das Problem sind (Colomer und Magalhaes 2012). Angesichts der Umstände können jedoch häufig die neuen Regierungen kaum etwas anderes tun als die vorherigen Regierungen - und dies wurde dramatisch deutlich bei den Regierungswechseln in Portugal, Spanien, Irland und Griechenland in den Jahren 2011 und 2012 (Armingeon und Baccaro 2012). Das Ausbleiben dieser neuen Koalitionen liegt wahrscheinlich daran, dass heute deren Voraussetzungen nicht mehr in dem Masse gegeben sind, wie früher: Politik war zu einem beträchtlichen Teil die Interessenpolitik sozialer Klassen und Gruppen, die eine kollektive Identität aufwiesen und durch Parteien und Verbände repräsentiert wurden. Heute sind sozialen Lagen heterogen, die Klassenzugehörigkeit hat eine nachlassende Prägekraft für politische Einstellungen und belastbare Beziehungen zwischen Bürgern und Parteien und Verbänden sind erodiert.

(2) Keine neuen Ideen. Während den großen historischen Krisen gab es einen Wettkampf der Ideen: Freihandel versus Protektionismus; Wohlfahrtsstaat oder liberaler Nachtwächterstaat; Demokratie oder Diktatur; makro-ökonomische Steuerung versus inflationsvermeidende Geldpolitik. Im Zeitraum seit 2007 gibt es - zumindest bislang - keine vergleichbaren Kontroversen. Nach dem kurzen keynesianischen Strohfeuer in einigen Ländern zwischen 2008 und 2010 setzte jede Regierung auf das Ziel der fiskalischen Konsolidierung und auf Austerität als Mittel. Solange eine Wachstumsphase ausbleibt, die politischen Spielraum schaffen könnte, ist die Idee des Sparens konkurrenzlos.

(3) Keine Krise der bestehenden politischen Ordnung. Im späten 19. Jahrhundert und in den 1930er Jahren hatten Wirtschaftskrisen eine direkte Wirkung auf die Stabilität der 
politischen Ordnung. Seit 2007 gibt es bislang keine Zeichen einer schwerwiegenden Instabilität der Demokratie. Zwar gewinnen xenophobe und rechtspopulistische Parteien vermehrt Stimmen bei nationalen Wahlen, aber nichts deutet derzeit darauf hin, dass die demokratische Ordnung zur Debatte steht. Eine der grundlegenden Lektionen der Weltwirtschaftskrise in den 1930er Jahren war die Erkenntnis, dass ein ausgebauter Wohlfahrtsstaat eine Fortsetzung der wirtschaftlichen in einer politischen Krise verhindern kann. Diese stabilisierende Rolle des Wohlfahrtsstaats hat sich in den vergangen fünf Jahren gezeigt. Eine Konsequenz der früheren Krisen - der Ausbau des Wohlfahrtsstaates - kann erklären, weshalb dieses Mal die ökonomische Krise nicht in eine politische Krise mündete.

(4) Keine greifbaren Optionen eines neuen Wohlfahrtsstaates. In einer Studie über neue soziale Risiken nutzte Bonoli (Bonoli 2007) Piersons Idee von 'policies in time' und argumentierte, dass diese neuen sozialen Politiken schwierig zu verabschieden und zu implementieren seien, solange der vorhandene Spielraum für Politiken bereits nahezu vollständig durch, alte Sozialpolitiken’ (z.B. generöse Rentensysteme) besetzt sei. Dies scheint gegenwärtig der Fall zu sein. Bei den jüngsten sozialpolitischen Reformen geht es vor allem um eine Reduktion der Kosten der bisherigen Programme. Ich konnte keine qualitativen oder quantitativen Belege finden, dass Politikerinnen und Politiker die Krise nutzen, um Ressourcen von den alten Politiken auf die ,neuen’ Sozialpolitiken zu verschieben. Vielmehr versuchten sie einfach Kosten zu sparen, wo Kosten gespart werden konnten. Wie das extreme Beispiel der südeuropäischen Länder zeigt, ist es für Regierungen schon schwierig genug, auf dem Grat der Akzeptanz durch EU und IMF auf der einen Seite und den Wiederwahlnotwendigkeiten in nationalen Wahlkämpfen auf der anderen Seite zu balancieren. Unter diesen Bedingungen der vielfachen Handlungseinschränkungen wird ein Umbau und eine Modernisierung des Wohlfahrtsstaates zu einer unerfüllbaren Herausforderung.

(5) Kein fiskalischer Handlungsspielraum aufgrund früherer politischer Entscheidungen. Der fiskalische Handlungsspielraum ist nicht nur aufgrund des breiten Konsenses über Notwendigkeit einer fiskalischen Konsolidierung schwerwiegend eingeschränkt. Darüber hinaus hat die Entscheidung zur Einführung der Währungsunion mit ihren Implikationen viele frühere Handlungsmöglichkeiten von Regierungen beseitigt (siehe auch Scharpf in diesem Band). Politikoptionen wie eine Abwertung der nationalen Währung oder eine entschiedene anti-zyklische Politik sind institutionell beseitigt. In 
dieser Hinsicht war Politik tatsächlich für die Krisenpolitik bestimmend: Freilich nicht im Sinne eines parteipolitischen Wettbewerbs über die besten Lösungsmöglichkeiten, sondern im Sinne eines Kompromisses der Regierungen in Maastricht, welcher für nachfolgende Parteienregierungen den Handlungsspielraum dramatisch einschränkte.

Folgt daraus wirklich, dass alle demokratischen und wirtschaftlich entwickelten Nationen auf ein Austeritätsregime mit einem teilweise abgebauten Wohlfahrtsstaat konvergieren? Es gibt mindestens drei Gegenargumente: (1) Mit aller Wahrscheinlichkeit werden Politiker große Probleme haben die Austeritätsprogramme umzusetzen. Diese Implementationsfähigkeit wird aufgrund unseres bisherigen Wissens stark von Institutionen geprägt, wie Regierungssysteme (Alesina 2010), die Position von Finanzministern in Kabinetten (Hallerberg und Hagen 1999; Hallerberg et al. 2009) und wohlfahrtsstaatliche Strukturen (Wagschal und Wenzelburger 2008, 2012). Das gleiche Austeritätsprogramm wird nach seiner Implementierung in zwei Nationen voraussichtlich sehr unterschiedliche Effekte haben. (2) Ein erheblicher Teil der großen Veränderungen von Politiken geht nicht auf große, zeitlich limitierte Reformen zurück, sondern ergibt sich als Resultat von kleinen, inkrementellen und akkumulierten Änderungen über eine lange Zeitspanne (Thelen 2003; Streeck und Thelen 2005). Wahrscheinlich werden die Austeritätsprogramme in sehr vielen kleinen und leicht unterschiedlichen Schritten umgesetzt, deren Gesamteffekt zu beträchtlichen Unterschieden zwischen Nationen führen könnte. Der zukünftige Wohlfahrtsstaat des Austeritätszeitalters wird voraussichtlich national stark variieren. Es wird jedoch schwierig sein, diese Variationen auf unterschiedliche parteipolitische Programme zurückzuführen. (3) Das Zusammenspiel von Institutionen und wirtschaftliche Leistungsfähigkeiten könnte $\mathrm{zu}$ sehr unterschiedlichen fiskalischen Leistungsprofilen und damit auch zu unterschiedlichen wohlfahrtsstaatlichen Optionen führen. Beispielsweise könnten die von EU und IMF aufgezwungenen Austeritätspläne in Griechenland zusammen mit der beseitigten Abwertungsoption zu niedrigem Wachstum in diesem Lande führen. Hingegen ist die Mitgliedschaft in der Euro-Zone eine wichtige Voraussetzung für das, durch den Export getragene, Wirtschaftswachstum und die dadurch generierten Ressourcen für neuen Politiken in Deutschland (Armingeon und Baccaro 2012; Flassbeck 2012; IMF 2012).

\section{Anhang}


Tabelle 3 (Anhang): Regression Zinssatz (FE Jahresdummies)

\begin{tabular}{|c|c|c|c|c|c|}
\hline & $\begin{array}{r}\text { Zinssatz } \\
\text { Modell } 1 \\
\end{array}$ & $\begin{array}{c}\text { Zinssatz } \\
\text { Modell } 2 \\
\end{array}$ & $\begin{array}{c}\text { Zinssatz } \\
\text { Modell } 3 \\
\end{array}$ & $\begin{array}{c}\text { Zinssatz } \\
\text { Modell } 4 \\
\end{array}$ & $\begin{array}{r}\text { Zinssatz } \\
\text { Modell } 5 \\
\end{array}$ \\
\hline$\overline{\text { Zinssatz_Lag1 }}$ & $\begin{array}{l}0.867 \\
(0.116)^{* * * *}\end{array}$ & $\begin{array}{l}0.849 \\
(0.099)^{* * * *}\end{array}$ & $\begin{array}{l}0.916 \\
(0.119)^{* * * *}\end{array}$ & $\begin{array}{l}0.859 \\
(0.105)^{* * * *}\end{array}$ & $\begin{array}{l}0.859 \\
(0.105)^{* * * *}\end{array}$ \\
\hline Zinssatz_Lag2 & $\begin{array}{r}-0.042 \\
(0.121)\end{array}$ & $\begin{array}{l}-0.058 \\
(0.098)\end{array}$ & $\begin{array}{l}-0.110 \\
(0.101)\end{array}$ & $\begin{array}{l}-0.051 \\
(0.108)\end{array}$ & $\begin{array}{l}-0.051 \\
(0.108)\end{array}$ \\
\hline Schulden & $\begin{array}{r}0.000 \\
(0.002)\end{array}$ & $\begin{array}{r}0.000 \\
(0.002)\end{array}$ & $\begin{array}{l}-0.001 \\
(0.002)\end{array}$ & $\begin{array}{r}0.000 \\
(0.002)\end{array}$ & $\begin{array}{r}0.000 \\
(0.002)\end{array}$ \\
\hline Schulden_Trend & $\begin{array}{l}-0.006 \\
(0.005)\end{array}$ & $\begin{array}{l}-0.010 \\
(0.005)^{* *}\end{array}$ & $\begin{array}{l}-0.005 \\
(0.004)\end{array}$ & $\begin{array}{l}-0.010 \\
(0.005)^{* *}\end{array}$ & $\begin{array}{l}-0.010 \\
(0.005)^{* *}\end{array}$ \\
\hline Saldo & $\begin{array}{r}-0.010 \\
(0.023)\end{array}$ & $\begin{array}{l}-0.026 \\
(0.017)^{*}\end{array}$ & $\begin{array}{l}-0.015 \\
(0.014)\end{array}$ & $\begin{array}{l}-0.030 \\
(0.017)^{* *}\end{array}$ & $\begin{array}{l}-0.030 \\
(0.017)^{* *}\end{array}$ \\
\hline Wirtschaftswachstum & $\begin{array}{r}0.025 \\
(0.032)\end{array}$ & $\begin{array}{r}0.035 \\
(0.033)\end{array}$ & $\begin{array}{r}0.034 \\
(0.029)\end{array}$ & $\begin{array}{r}0.026 \\
(0.035)\end{array}$ & $\begin{array}{r}0.026 \\
(0.035)\end{array}$ \\
\hline Regierungswechsel & & $\begin{array}{l}0.087 \\
(0.043)^{* * *}\end{array}$ & $\begin{array}{l}0.125 \\
(0.046)^{* * * *}\end{array}$ & $\begin{array}{l}0.123 \\
(0.054)^{* * *}\end{array}$ & $\begin{array}{l}0.123 \\
(0.054)^{* * *}\end{array}$ \\
\hline Gov_New & & $\begin{array}{r}0.105 \\
(0.118)\end{array}$ & $\begin{array}{l}0.007 \\
(0.068)\end{array}$ & & \\
\hline Föderalismus & & $\begin{array}{r}-0.045 \\
(0.126)\end{array}$ & $\begin{array}{r}-0.015 \\
(0.094)\end{array}$ & & \\
\hline Stabile Koalition & & $\begin{array}{r}0.012 \\
(0.086)\end{array}$ & $\begin{array}{l}0.021 \\
(0.090)\end{array}$ & & \\
\hline $\begin{array}{l}\text { Wohlfahrtsstaat } \\
\text { (Ausmaß) }\end{array}$ & & $\begin{array}{l}-0.036 \\
(0.013)^{* * * *}\end{array}$ & $\begin{array}{l}-0.025 \\
(0.013)^{* *}\end{array}$ & $\begin{array}{l}-0.032 \\
(0.012)^{* * *}\end{array}$ & $\begin{array}{l}-0.032 \\
(0.012)^{* * *}\end{array}$ \\
\hline Staatseinnahmen & & $\begin{array}{c}0.018 \\
(0.011)^{*}\end{array}$ & $\begin{array}{l}0.018 \\
(0.008)^{* * *}\end{array}$ & $\begin{array}{l}0.018 \\
(0.008)^{* * *}\end{array}$ & $\begin{array}{l}0.018 \\
(0.008)^{* * *}\end{array}$ \\
\hline Gov_New_Gap & & $\begin{array}{l}-0.007 \\
(0.061)\end{array}$ & $\begin{array}{r}0.062 \\
(0.048)\end{array}$ & & \\
\hline$\Delta$ Staatssektor & & $\begin{array}{l}0.072 \\
(0.035)^{* * *}\end{array}$ & $\begin{array}{l}0.074 \\
(0.038)^{* *}\end{array}$ & $\begin{array}{l}0.091 \\
(0.033)^{* * * *}\end{array}$ & $\begin{array}{l}0.091 \\
(0.033) * * * *\end{array}$ \\
\hline$\Delta$ Wohlfahrtsstaat & & $\begin{array}{r}0.149 \\
(0.125)\end{array}$ & $\begin{array}{r}0.048 \\
(0.048)\end{array}$ & & \\
\hline Rechtsregierung & & $\begin{array}{l}-0.001 \\
(0.001)^{* * *}\end{array}$ & $\begin{array}{l}-0.001 \\
(0.001)^{* *}\end{array}$ & $\begin{array}{l}-0.001 \\
(0.000)^{* * *}\end{array}$ & $\begin{array}{l}-0.001 \\
(0.000)^{* * *}\end{array}$ \\
\hline Streik_Lag1 & & & $\begin{array}{l}0.000 \\
(0.000)^{* * * *}\end{array}$ & & \\
\hline Konstante & $\begin{array}{r}0.196 \\
(0.269)\end{array}$ & $\begin{array}{r}-0.340 \\
(0.583)\end{array}$ & $\begin{array}{r}-0.425 \\
(0.567)\end{array}$ & $\begin{array}{l}-0.201 \\
(0.521)\end{array}$ & $\begin{array}{l}-0.201 \\
(0.521)\end{array}$ \\
\hline $\begin{array}{l}\mathrm{R}^{2} \\
\mathrm{~N}\end{array}$ & $\begin{array}{c}0.84 \\
792\end{array}$ & $\begin{array}{c}0.86 \\
760\end{array}$ & $\begin{array}{c}0.89 \\
677\end{array}$ & $\begin{array}{c}0.86 \\
761\end{array}$ & $\begin{array}{c}0.86 \\
761\end{array}$ \\
\hline
\end{tabular}

Anmerkungen: Koeffizienten der Jahresdummies werden nicht berichtet. $*^{* * *} p<.01$; ${ }^{* * *} p<.05$; $*^{* *} p<.10 ;{ }^{*} p<.15$. Standardfehler in Klammern.

Tabelle 4 (Anhang): Regression Zinssatz (Erste Differenzen)

\begin{tabular}{llllll}
\hline & $\Delta$ Zinssatz & $\Delta$ Zinssatz & $\Delta$ Zinssatz & $\Delta$ Zinssatz & $\Delta$ Zinssatz \\
& Modell 1 & Modell 2 & Modell 3 & Modell 4 & Modell 5 \\
\hline Zinssatz_Lag2 & -0.150 & -0.173 & -0.174 & -0.170 & -0.164 \\
& $(0.034)^{* * * *}$ & $(0.032)^{* * * *}$ & $(0.031)^{* * * *}$ & $(0.031)^{* * * *}$ & $(0.036)^{* * * *}$ \\
Schulden & -0.001 & -0.000 & -0.002 & -0.000 & -0.001 \\
& $(0.002)$ & $(0.002)$ & $(0.001)$ & $(0.002)$ & $(0.002)$ \\
Schulden_Trend & -0.007 & -0.005 & -0.003 & -0.004 & -0.007 \\
& $(0.007)$ & $(0.005)$ & $(0.004)$ & $(0.005)$ & $(0.007)$
\end{tabular}




\begin{tabular}{|c|c|c|c|c|c|}
\hline Saldo & $\begin{array}{l}-0.039 \\
(0.023)^{*}\end{array}$ & $\begin{array}{l}-0.040 \\
(0.020)^{* * *}\end{array}$ & $\begin{array}{l}-0.035 \\
(0.017)^{* * *}\end{array}$ & $\begin{array}{l}-0.038 \\
(0.019)^{* * *}\end{array}$ & $\begin{array}{l}-0.055 \\
(0.023)^{* * *}\end{array}$ \\
\hline Wirtschaftswachstum & $\begin{array}{r}0.015 \\
(0.032)\end{array}$ & $\begin{array}{c}0.029 \\
(0.025)\end{array}$ & $\begin{array}{r}0.027 \\
(0.024)\end{array}$ & $\begin{array}{r}0.030 \\
(0.025)\end{array}$ & $\begin{array}{r}0.019 \\
(0.035)\end{array}$ \\
\hline Gov_Change & & $\begin{array}{c}0.056 \\
(0.036)^{*}\end{array}$ & $\begin{array}{c}0.075 \\
(0.046)^{*}\end{array}$ & $\begin{array}{c}0.061 \\
(0.041)^{*}\end{array}$ & $\begin{array}{l}0.095 \\
(0.051)^{* *}\end{array}$ \\
\hline Gov_New & & $\begin{array}{r}-0.004 \\
(0.084)\end{array}$ & $\begin{array}{r}-0.078 \\
(0.062)\end{array}$ & & \\
\hline Föderalismus & & $\begin{array}{r}-0.034 \\
(0.094)\end{array}$ & $\begin{array}{r}-0.044 \\
(0.088)\end{array}$ & & \\
\hline Stabile Koalition & & $\begin{array}{r}-0.010 \\
(0.086)\end{array}$ & $\begin{array}{r}-0.013 \\
(0.086)\end{array}$ & & \\
\hline $\begin{array}{l}\text { Wohlfahrtsstaat } \\
\text { (Ausmaß) }\end{array}$ & & $\begin{array}{l}-0.030 \\
(0.009) * * * *\end{array}$ & $\begin{array}{l}-0.020 \\
(0.010)^{* *}\end{array}$ & $\begin{array}{l}-0.030 \\
(0.008) * * * *\end{array}$ & $\begin{array}{l}-0.023 \\
(0.010)^{* * *}\end{array}$ \\
\hline Staatseinnahmen & & $\begin{array}{l}0.023 \\
(0.008)^{* * *}\end{array}$ & $\begin{array}{l}0.021 \\
(0.006)^{* * * *}\end{array}$ & $\begin{array}{l}0.023 \\
(0.007)^{* * * *}\end{array}$ & $\begin{array}{l}0.023 \\
(0.007)^{* * * *}\end{array}$ \\
\hline Gov_New_Gap & & $\begin{array}{r}-0.018 \\
(0.048)\end{array}$ & $\begin{array}{r}0.045 \\
(0.040)\end{array}$ & & \\
\hline$\Delta$ Staatssektor & & $\begin{array}{c}0.044 \\
(0.028)^{*}\end{array}$ & $\begin{array}{l}0.066 \\
(0.027)^{* * *}\end{array}$ & $\begin{array}{c}0.043 \\
(0.028)^{*}\end{array}$ & \\
\hline$\Delta$ Wohlfahrtsstaat & & $\begin{array}{c}0.225 \\
(0.148)^{*}\end{array}$ & $\begin{array}{r}0.100 \\
(0.081)\end{array}$ & $\begin{array}{c}0.227 \\
(0.150)^{*}\end{array}$ & \\
\hline Rechtsregierung & & $\begin{array}{r}-0.001 \\
(0.001)\end{array}$ & $\begin{array}{r}-0.001 \\
(0.001)\end{array}$ & $\begin{array}{l}-0.001 \\
(0.001)^{*}\end{array}$ & \\
\hline Streik_Lag1 & & & $\begin{array}{l}0.000 \\
(0.000)^{* * * *}\end{array}$ & & \\
\hline Konstante & $\begin{array}{r}0.163 \\
(0.314)\end{array}$ & $\begin{array}{r}-0.504 \\
(0.523)\end{array}$ & $\begin{array}{r}-0.547 \\
(0.439)\end{array}$ & $\begin{array}{r}-0.543 \\
(0.448)\end{array}$ & $\begin{array}{r}-0.533 \\
(0.527)\end{array}$ \\
\hline$\overline{\mathrm{R}^{2}}$ & 0.13 & 0.19 & 0.31 & 0.18 & 0.14 \\
\hline $\mathrm{N}$ & 792 & 760 & 677 & 761 & 763 \\
\hline
\end{tabular}

Anmerkung: ${ }^{* * * *} p<.01 ; * * * p<.05 ;{ }^{* *} p<.10 ;{ }^{*} p<.15$. Standardfehler in Klammern.

Tabelle 5 (Anhang): Signifikanzen der politischen Variablen in Abhängigkeit von Modellspezifikation und Signifikanzniveau

\begin{tabular}{lccccccccc}
\hline Spezifikation & LDV & FE & Dif & LDV & FE & Dif & LDV & FE & Dif \\
Signifikanzniveau. & $15 \%$ & $15 \%$ & $15 \%$ & $10 \%$ & $10 \%$ & $10 \%$ & $5 \%$ & $5 \%$ & $5 \%$ \\
\hline Regierungswechsel & $\mathrm{x}$ & $\mathrm{x}$ & $\mathrm{x}$ & & $\mathrm{x}$ & & & $\mathrm{x}$ & \\
Ausmass & & & & & & & & & \\
Wohlfahrtsstaat & $\mathrm{x}$ & $\mathrm{x}$ & $\mathrm{x}$ & $\mathrm{x}$ & $\mathrm{x}$ & $\mathrm{x}$ & $\mathrm{x}$ & $\mathrm{x}$ & $\mathrm{x}$ \\
Ausmass Staatssektor & $\mathrm{x}$ & $\mathrm{x}$ & $\mathrm{x}$ & $\mathrm{x}$ & $\mathrm{x}$ & $\mathrm{x}$ & $\mathrm{x}$ & $\mathrm{x}$ & $\mathrm{x}$ \\
$\Delta$ Staatssektor & $\mathrm{x}$ & $\mathrm{x}$ & $\mathrm{x}$ & $\mathrm{x}$ & $\mathrm{x}$ & & & $\mathrm{x}$ & \\
$\Delta$ Wohlfahrtsstaat & $\mathrm{x}$ & & $\mathrm{x}$ & & & & & & \\
Rechtsregierung & $\mathrm{x}$ & $\mathrm{x}$ & $\mathrm{x}$ & & $\mathrm{x}$ & & & $\mathrm{x}$ & \\
Streik & $\mathrm{x}$ & $\mathrm{x}$ & $\mathrm{x}$ & $\mathrm{x}$ & $\mathrm{x}$ & $\mathrm{x}$ & $\mathrm{x}$ & $\mathrm{x}$ & $\mathrm{x}$ \\
\hline
\end{tabular}


Basis: Tabellen 2-4. LDV: Modell mit zeitverzögerten Variablen; FE: Modelle mit fixed effects (Zeit). Dif: Modelle mit ersten Differenzen $\left(t_{n}-t_{n-1}\right)$ des Zinssatzes .

\section{References}

Alesina, Alberto. 2010. Fiscal Adjustments: Lessons from Recent History. Cambridge, Mass. (Harvard University): Unpublished paper, prepared for Ecofin meeting, Madrid, April 15, 2010.

Armingeon, Klaus. 2002. The Effects of Negotiation Democracy. A Comparative Analysis. European Journal of Political Research 41: 81-105.

Armingeon, Klaus. 2012. The Politics of Fiscal Responses to the Crisis of 2008-2009. Governance 25(4): 543-565.

Armingeon, Klaus und Lucio Baccaro. 2012. The Sorrows of Young Euro: Policy Responses to the Sovereign Debt Crisis. In Coping with Crisis: Government Reactions to the Great Recession, Hrsg. Nancy Bermeo und Jonas Pontusson, 162-197. New York: Russel Sage.

Armingeon, Klaus und Giuliano Bonoli (Hrsg.). 2006. The Politics of Postindustrial Welfare States. Adapting Postwar Social Policies to New Social Risks. London: Routledge.

Armingeon, Klaus, Romana Careja, David Weisstanner, Sarah Engler, Panajotis Potolidis, Marlène Gerber und Philipp Leimgruber. 2012. Comparative Political Data Set III 1990-2010. Bern: Institute of Political Science, University of Berne.

Armingeon, Klaus, David Weisstanner, Sarah Engler, Panajotis Potolidis, Marlène Gerber und Phillipp Leimgruber. 2011. Comparative Political Data Set I 1960-2009. Bern: Institute of Political Science, University of Berne.

Baltagi, Badi H. 2010. Econometric Analysis of Panel Data, Fourth Edition. Chichester: John Wiley and Sons.

Beck, Nathaniel und Jonathan Katz. 2011. Modeling Dynamics in Time-Series-Cross-Section Political Economy Data. Annual Review of Political Science 14: 331-52.

Blanchard, Olivier. 2012. 2011 in Review: Four Hard Truths. http://blogimfdirect.imf.org/2011/12/21/2011-in-review-four-hard-truths/ (06.08.2012). 
Bonoli, Giuliano. 2007. Time Matters: Postindustrialization, New Social Risks, and Welfare State Adaptation in Advanced Industrial Democracies. Comparative Political Studies 40: 495-520.

Cameron, David R. 2012. Fiscal Responses to the Economic Contraction of 2008-09. In Coping with Crisis: Government Reactions to the Great Recession, Hrsg. Nancy Bermeo und Jonas Pontusson, 91-129. New York: Russell Sage.

Caramani, Daniele, Kevin Deegan-Krause und Rainbow Murray. 2011. Political Data in 2010. European Journal of Political Research 50: 869-87.

Castles, Francis G. 2010. Black Swans and Elephants on the Move: The Impact of Emergencies on the Welfare State. Journal of European Social Policy 20: 91-101.

Colomer, Josep M. und Pedro Magalhaes. 2012. Firing the Coach: How Governments Are Losing Elections in Europe. Washington, D.C.: Paper prepared for the conference 'Political Consequences of the Economic Crisis: Voting and Protesting in Europe', Georgetown University, April 2012.

Esping-Andersen, Gosta. 1990. The Three Worlds of Welfare Capitalism. Princeton: Princeton University Press.

Flassbeck, Heiner. 2012. Zehn Mythen der Krise. Frankfurt am Main: Suhrkamp.

Gale, William G. und Peter R. Orszag. 2003. Economic Effects of Sustained Budget Deficits. National Tax Journal 56: 463-85.

Gärtner, Manfred und Björn Griesbach. 2012. Rating Agencies, Self-Fulfilling Prophecy and Multiple Equilibria? An Empirical Model of the European Sovereign Debt Crisis 2009-2011. June 2012 Discussion Paper No. 2012-15. St. Gallen: University of St. Gallen, Department of Economics.

Gourevitch, Peter. 1986. Politics in Hard Times. Comparative Responses to International Economic Crises. Ithaca/London: Cornell University Press.

Hall, Peter A. 2013. The Political Origins of Our Economic Discontents: Contemporary Adjustment Problems in Historical Perspective. In Politics in the New Hard Times, Hrsg. Miles Kahler und David Lake. Ithaca: Cornell University Press.

Hall, Peter A. und David Soskice (Hrsg.). 2001. Varieties of Capitalism. The Institutional Foundations of Comparative Advantage. Oxford: Oxford University Press.

Hallerberg, Mark und Jürgen von Hagen. 1999. Electoral Institutions, Cabinet Negotiations, and Budget Deficits within the European Union. In Fiscal Institutions and Fiscal Performance, Hrsg. James Poterba und Jürgen von Hagen, 209-232. Chicago: Chicago University Press. 
Hallerberg, Mark, Rolf Rainer Strauch und Jürgen von Hagen. 2009. Fiscal Governance in Europe. Cambridge: Cambridge University Press.

ILO und World Bank. 2012. Inventory of Policy Responses to the Financial and Economic Crisis. Geneva und Washington, D.C.: ILO und World Bank.

IMF. 2011a. Fiscal Monitor. Adressing Fiscal Challenges to Reduce Economic Risks. September 2011. Washington, D.C.: IMF.

IMF. 2012. Fiscal Monitor. Balancing Fiscal Policy Risks. April 2012. Washington, D.C.: IMF.

IMF. 2010a. Fiscal Monitor. Fiscal Exit. From Strategy to Implementation. November 2010. Washington, D.C.: IMF.

IMF. 2011b. Fiscal Monitor. Shifting Gears. Tackling Challenges on the Road to Fiscal Adjustment. April 2011. Washington, D.C.: IMF.

IMF. 2010b. From Stimulus to Consolidation: Revenue and Expenditure Policies in Advanced and Emerging Economies. Washington, D.C.: IMF.

Jaramillo, Laura und Anke Weber. 2012. Bond Yields in Emerging Economies: It Matters What State You Are in (IMF Working Paper/12/198). Washington, D.C.: IMF.

Katzenstein, Peter J. 2003. Small States and Small States Revisited. New Political Economy 8: 9-30.

Katzenstein, Peter J. 1985. Small States in World Markets. Industrial Policy in Europe. Ithaca/London: Cornell University Press.

Kingdon, John W. 1984. Agendas, Alternatives, and Public Policies. Boston/Toronto: Harper Collins.

Laubach, Thomas. 2009. New Evidence on the Interest Rate Effect of Budget Deficits and Debt. Journal of the European Economic Association 7: 858-85.

Lijphart, Arend. 1999. Patterns of Democracy: Government Form and Performance in ThirtySix Countries. New Haven: Yale University Press.

Lindvall, Johannes. 2012. Policies and Politics in Two Economic Crises. In Coping with Crisis: Government Reaction to the Great Recession, Hrsg. Nancy Bermeo und Jonas Pontusson, 233-260. New York: Russel Sage.

Mares, Isabela. 2010. Macroeconomic Outcomes. In Oxford Handbook on Welfare States, Hrsg. Francis Castles, Stephan Leibfried, Jane Lewis, Herbert Obinger und Christopher Pierson, 539-51. Oxford: Oxford University Press.

Obinger, Herbert. 2004. Politik und Wirtschaftswachstum. Ein internationaler Vergleich. Wiesbaden: Verlag für Sozialwissenschaften. 
OECD. 2012. Economic Policy Reforms 2012: Going for Growth. Paris: OECD.

Pierson, Paul. 1994. Dismantling the Welfare State? Reagan, Thatcher, and the Politics of Retrenchment. Cambridge: Cambridge University Press.

Pontusson, Jonas. 2005. Inequality and Prosperity. Social Europe Vs. Liberal America. Ithaca und London: Cornell University Press.

Scharpf, Fritz W. 1988. The Joint Decision Trap: Lessons from German Federalism and European Integration. Public Administration 66: 239-78.

Schmidt, Manfred G. 2010a. Demokratietheorien. Eine Einführung, 5. Aufl. Wiesbaden: Verlag für Sozialwissenschaften.

Schmidt, Manfred G. 2010b. Parties. In Oxford Handbook on Welfare States, Hrsg. Francis Castles, Stephan Leibfried, Jane Lewis, Herbert Obinger und Christopher Pierson, 211-26. Oxford: Oxford University Press.

Schmidt, Manfred G. 1996. When Parties Matter: A Review of the Possibilities and Limits of Partisan Influence on Public Policy. European Journal of Political Research 30: 15583.

Schmidt, Manfred G. 2012. Wirklich nur Mittelmaß? Deutschlands Sozialstaat im Spiegel neuer, international vergleichender Daten. Zeitschrift für Staats- und Europawissenschaften 10: 159-95.

Streeck, Wolfgang und Kathleen Thelen. 2005. Introduction: Institutional Chance in Advanced Political Economies. In Beyond Continuity. Institutional Change in Advanced Political Economies, Hrsg. Wolfgang Streeck und Kathleen Thelen, 1-39. Oxford: Oxford University Press.

Thelen, Kathleen. 2003. How Institutions Evolve: Insights from Comparative-Historical Analysis. In Comparative Historical Analysis in the Social Sciences, Hrsg. James Mahoney und Dietrich Rueschemeyer, 208-40. New York: Cambridge University Press.

Wagschal, Uwe und Georg Wenzelburger. 2008. Roads to Success: Budget Consolidations in Oecd Countries. Journal of Public Policy 28: 309-39.

Wagschal, Uwe und Georg Wenzelburger. 2012. When Do Governments Consolidate? A Quantitative Comparative Analysis of 23 OECD Countries (1980-2005). Journal of Comparative Policy Analysis: Research and Practice 14: 45-71. 